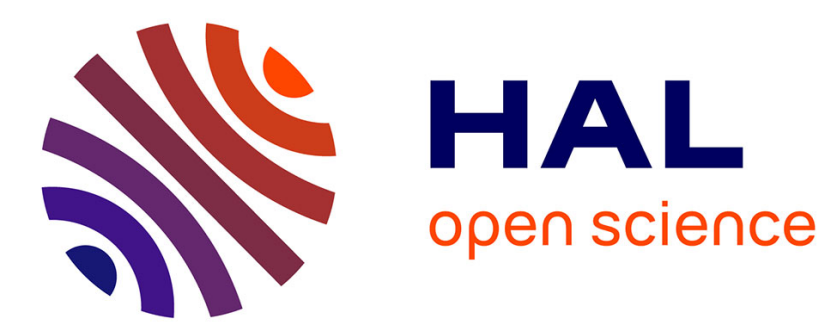

\title{
On the Poleward Motion of Midlatitude Cyclones in a Baroclinic Meandering Jet
}

\author{
Ludivine Oruba, Guillaume Lapeyre, Gwendal Rivière
}

\section{To cite this version:}

Ludivine Oruba, Guillaume Lapeyre, Gwendal Rivière. On the Poleward Motion of Midlatitude Cyclones in a Baroclinic Meandering Jet. Journal of the Atmospheric Sciences, 2013, 70 (8), pp.26292649. 10.1175/jas-d-12-0341.1 . hal-01099074

\section{HAL Id: hal-01099074 https://hal.science/hal-01099074}

Submitted on 1 Jan 2015

HAL is a multi-disciplinary open access archive for the deposit and dissemination of scientific research documents, whether they are published or not. The documents may come from teaching and research institutions in France or abroad, or from public or private research centers.
L'archive ouverte pluridisciplinaire HAL, est destinée au dépôt et à la diffusion de documents scientifiques de niveau recherche, publiés ou non, émanant des établissements d'enseignement et de recherche français ou étrangers, des laboratoires publics ou privés. 


\title{
On the Poleward Motion of Midlatitude Cyclones in a Baroclinic Meandering Jet
}

\author{
LUDIVINE ORUBA AND GUILLAUME LAPEYRE \\ Laboratoire de Météorologie Dynamique/IPSL, ENS/CNRS/UPMC, Paris, France
}

GWENDAL RIVIÈRE

CNRM/GAME, Météo-France, and CNRS, Toulouse, France

(Manuscript received 10 December 2012, in final form 6 March 2013)

\begin{abstract}
The motion of surface depressions evolving in a background meandering baroclinic jet is investigated using a two-layer quasigeostrophic model on a beta plane. Synoptic-scale finite-amplitude cyclones are initialized in the lower and upper layer to the south of the jet in a configuration favorable to their baroclinic interaction. The lower-layer cyclone is shown to move across the jet axis from its warm-air to cold-air side. It is the presence of a poleward-oriented barotropic potential vorticity (PV) gradient that makes possible the cross-jet motion through the beta-drift mechanism generalized to a baroclinic atmospheric context.

The potential vorticity gradient associated with the jet is responsible for the dispersion of Rossby waves by the cyclones and the development of an anticyclonic anomaly in the upper layer. This anticyclone forms a PV dipole with the upper-layer cyclone that nonlinearly advects the lower-layer cyclone across the jet.

In addition, the background deformation is shown to modulate the cross-jet advection. Cyclones evolving in a deformation-dominated environment (south of troughs) are strongly stretched while those evolving in a rotation-dominated environment (south of ridges) remain quasi isotropic. It is shown that the more stretched cyclones trigger a more efficient dispersion of energy, create a stronger upper-layer anticyclone, and move perpendicularly to the jet faster than the less stretched ones. Both the intensity and location of the upper-layer anticyclone explain the distinct cross-jet speeds. A statistical study consisting in initializing cyclones at different locations south of the jet core confirms that the cross-jet motion is faster for the more meridionally elongated cyclones evolving in areas of strongest barotropic PV gradient.
\end{abstract}

\section{Introduction}

A commonly observed feature of midlatitude winter cyclones is their tendency to cross the axis of the climatological jet streams from the equatorward to the poleward side as deduced from case-to-case observational maps (Palmen and Newton 1969, see their Fig. 3.17), bandpass filtered atmospheric fields (Blackmon et al. 1977; Wallace et al. 1988), or Lagrangian-based automatic tracking algorithms (Hoskins and Hodges 2002; Neu et al. 2013). It fits with our common thinking about the life cycle of individual surface cyclones, which usually form along fronts located on the warm-air side of the instantaneous jet streams and move to their cold-air side during the occluding process (Vederman 1954; Palmen

Corresponding author address: Ludivine Oruba, LRA-ENS, 24 rue Lhomond, 75005 Paris, France.

E-mail: loruba@lra.ens.fr and Newton 1969; Blackmon et al. 1977). Since occlusion is the frontolytic process during which the surface low becomes surrounded by cold air, the cyclone center cannot stay below the thermal wind maximum during this latter stage and thus moves poleward relative to the jet to reach its cold-air zone. This qualitative vision does not preclude other interpretations that were provided in the midtwentieth century to explain the trajectory and deepening of surface cyclones according to their position relative to the instantaneous jet streams. As initially underlined by Namias and Clapp (1949) and Murray and Daniels (1953), the existence of a local jet creates a direct and an indirect transverse circulation at the jet entrance and exit, respectively. Owing to these ageostrophic transverse circulations, upper-level divergence appears at the right-entrance and left-exit regions of the jet that will tend to favor the rapid deepening of surface cyclones there. The left-exit regions of the jet streaksdefined as zones of local maxima in the instantaneous 
wind speed-were shown to be collocated with the rapid development phase of several winter cyclones [see Uccelini (1990) for a review]. However, this rule for the rapid-deepening growth stage of cyclones on the poleward side of a diffluent upper-level westerly flow cannot be considered as systematic, in particular when considering jets having a broader scale than jet streaks (Sanders 1993). Besides the previous observational studies, idealized nonlinear numerical simulations of extratropical cyclones show a clear poleward motion of surface lows relative to the jets as shown, for instance, by Simmons and Hoskins (1978) or Davies et al. (1991) from normalmode initialization or by Schär and Wernli (1993) from finite-amplitude anomaly initialization.

Recent observational campaigns have provided new insights on the properties of extratropical cyclones relative to jet streams. Most of the cyclones of the Fronts and Atlantic Storm Track Experiment (FASTEX) (Joly et al. 1999) campaign have revealed the occurrence of a rapid-deepening growth stage during the time interval when the storm crossed the jet axis, with or without the presence of a jet streak (Baehr et al. 1999). This was also observed for the December 1999 storm "Lothar" (Wernli et al. 2002), the January 2007 storm "Kyrill" (Fink et al. 2009), or the 26-28 February 2010 storm Xynthia (Rivière et al. 2012). As initially underlined by Rivière and Joly (2006a,b), it is the crossing of the large-scale slowly varying jet that seems to be a recurrent feature of eastern Atlantic storms. The slowly varying jet is associated with specific large-scale weather regimes and can be easily diagnosed by low-pass filtering (periods greater than 8 days) the atmospheric wind components. The aforementioned two papers identify different configurations in which the low-frequency jet-crossing phase may appear. Our goal is hereafter to study within an idealized framework the specific configuration analyzed in Rivière and Joly (2006a), which is illustrated by two examples in Fig. 1 using European Centre for MediumRange Weather Forecasts Interim Re-Analysis (ERAInterim) datasets. In both cases, the trajectory crosses the low-frequency jet close to its maximum wind speed (top panels). Both cyclones have already reached a large amplitude, are southwest-northeast elongated, and are located downstream of an upper-level disturbance when they are on the warm-air side of the jet (bottom panels). The case on the left side of Fig. 1 corresponds to one of the intensive observation period of the FASTEX campaign whose jet-crossing phase was detailed in Rivière and Joly (2006a) using FASTEX reanalysis data. It exhibits a slight transient decay phase before crossing the jet and rapidly deepens afterward. The case on the right side corresponds to the European storm Xynthia and deepens during the jet-crossing phase (Rivière et al. 2012).
Another common feature of these two cyclones is that the cyclone crosses the jet in the region where the jet changes its curvature from cyclonic to anticyclonic. This change in jet curvature is collocated with what was called a barotropic critical region in Rivière and Joly (2006a) and Rivière (2008). Such a region is defined from the effective deformation field, which is the difference between the square of the deformation magnitude and the square of the relative vorticity associated with the lowfrequency flow. A barotropic critical region is more precisely the separation area between two large-scale regions of positive effective deformation (red shadings in Fig. 1) located on both sides of the jet and having perpendicular dilatation axes. This can be also viewed as saddle points of the effective deformation field. Both cyclones crossed the jet close to the barotropic critical region (top panels) and rapidly contracted after the crossing (not shown). More generally, the large-scale deformation has a well-known effect on the stretching of surface cyclones and their associated frontal structures (Davies et al. 1991; Schultz et al. 1998; Wernli et al. 1998) but may also modulate their deepening rate and may constrain the location of their explosive growth stage (Rivière and Joly 2006a,b). The present paper addresses the role of the large-scale deformation associated with the low-frequency jet in the trajectory of surface cyclones. The answer to this question is particularly appealing since it could provide information on the trajectories of the cyclones from sole knowledge of the structure of the slowly varying environment and, thus, serve as a basis for predictability issues.

As previously mentioned, a few idealized numerical studies have been dedicated to understand the trajectory of midlatitude surface cyclones. Nevertheless, Gilet et al. (2009) proved the important role played by the vertically averaged meridional potential vorticity gradient (called barotropic PV gradient) and nonlinearities in the trajectory of midlatitude storms within a simple baroclinic environment (zonal jet). The underlying mechanism is related to the $\beta$-drift effect, which is well known in the context of tropical cyclones (e.g., Holland 1983) and oceanic eddies (e.g., McWilliams and Flierl 1979). Besides, the key role played by the large-scale barotropic PV gradient was confirmed in the real case of Xynthia (Rivière et al. 2012). Not only the environmental PV gradient matters but also the environmental deformation plays a role (Rivière 2008; Oruba et al. 2012). As shown in a barotropic quasigeostrophic context by Oruba et al. (2012), the deformation created by meandering westerly jets modulate the meridional displacement of a cyclonic eddy, which is primarily due to the $\beta$ drift. The deformation effects reinforce the anticyclone created by radiation of Rossby waves in the presence of a PV 

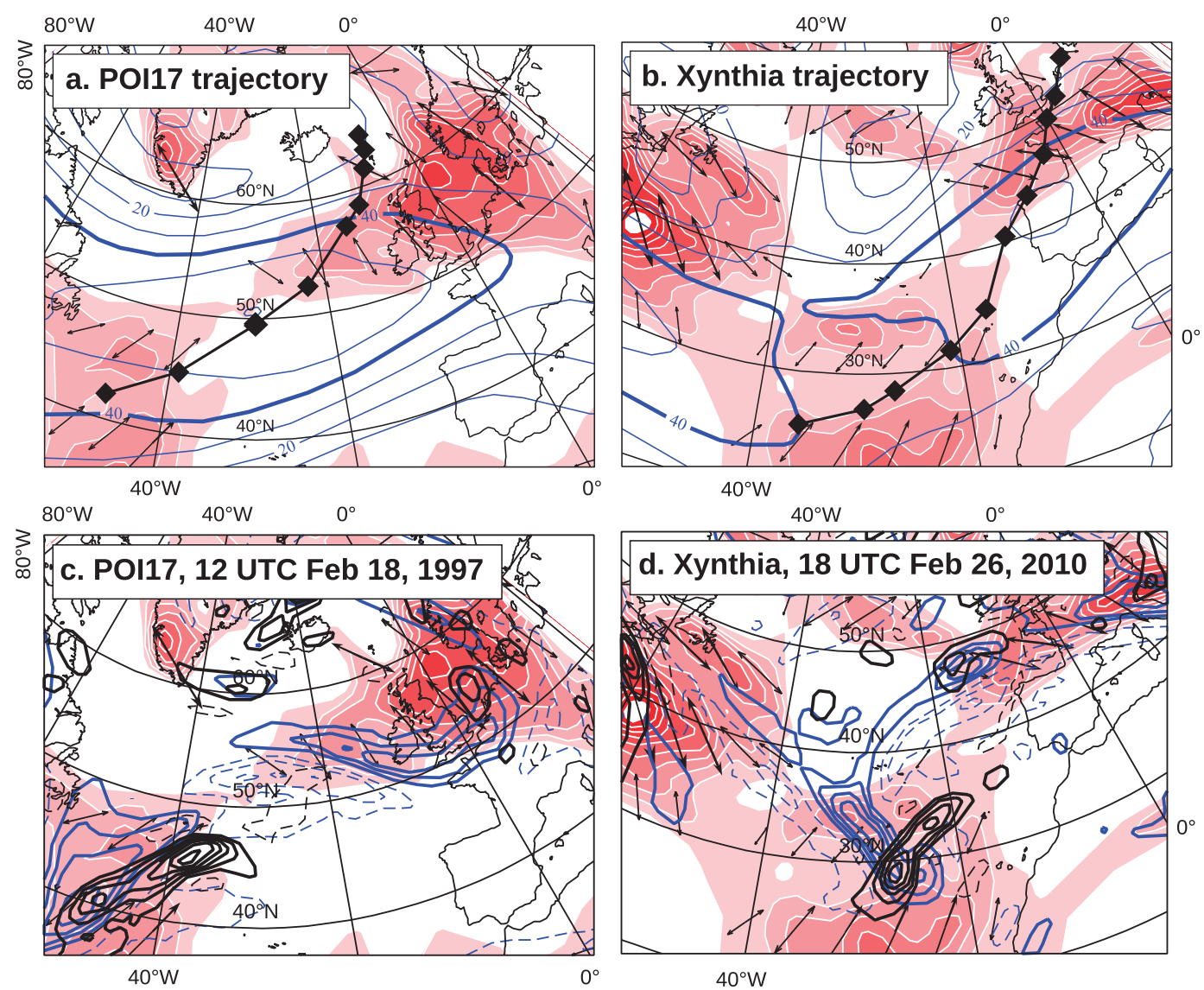

FIG. 1. Examples of crossing of the low-frequency jet by real extratropical cyclones for (left) the case of the intensive observation period 17 of FASTEX (18-20 Feb 1997) and (right) the storm Xynthia (26-28 Feb 2010). (top) Low-frequency wind speed (blue contours, interval $10 \mathrm{~m} \mathrm{~s}^{-1}$ ) (a) at 1200 UTC 18 Feb 1997 and (b) at 1800 UTC $26 \mathrm{Feb} 2010$, positive values of the low-frequency effective deformation (shaded red areas, interval $5 \times 10^{-10} \mathrm{~s}^{-2}$ ), and trajectory of the storms [black lines with diamonds corresponding to a 6-hourly time step (a) from 0600 UTC 18 Feb to 0600 UTC 20 Feb 1997 and (b) from 0600 UTC 26 Feb to 1800 UTC 28 Feb 2010]. (bottom) High-frequency relative vorticity at $300 \mathrm{hPa}$ (blue contours, interval $5 \times 10^{-5} \mathrm{~s}^{-1}$ ) and $900 \mathrm{hPa}$ (black contours, interval $5 \times 10^{-5} \mathrm{~s}^{-1}$ ) and effective deformation (same definition as in top panels). Arrows denote the dilatation axis.

gradient (which is the sum of the planetary vorticity gradient and the relative vorticity gradient in the presence of meridionally confined jets), and the newly created anticyclone interacts with the initial cyclone to form a dipole moving poleward. Whereas Gilet et al. (2009) considered zonal basic flows in a baroclinic context and Oruba et al. (2012) studied zonally inhomogeneous flows in a barotropic context, the present paper represents a step further by analyzing the same nonlinear $\beta$-drift mechanism for zonally inhomogeneous baroclinic flows. This attempt to characterize cyclone trajectories in meandering baroclinic flows should help us to gain more dynamical insights into the real cases shown in Fig. 1.

Several idealized studies on eddy displacement in a baroclinic atmosphere were made in the context of tropical cyclones and oceanic eddies. The effect of a vertically sheared environmental wind was studied by many authors
(Shapiro 1992; Wu and Emanuel 1993; Jones 1995, 2000a,b). Wu and Emanuel (1993) showed that it creates a vertical inclination of the structure, which allows a meridional displacement of the cyclone while it remains coherent thanks to a secondary circulation. The potential key role played by the PV gradient associated with the shear was mentioned by Shapiro (1992) but not deeply studied.

The trajectory of oceanic eddies in a baroclinic context was investigated by many authors through idealized studies in the absence (Mied and Lindemann 1979; Morel and McWilliams 1997; Sutyrin and Morel 1997; Lacasce 1998; Reznik and Kizner 2007) and presence of a large-scale current (Morel 1995; Vandermeirsch et al. 2001, 2003). In the presence of a vertically sheared largescale current, Vandermeirsch et al. (2001) showed that the effect of the baroclinic PV gradient associated with 
the shear that modifies the beta gyres is compensated by the advective effects of the environment [see also Morel (1995) for oceanic eddies and $\mathrm{Wu}$ and Emanuel (1993) for tropical cyclones]. As a result, the vortex is mainly advected by the planetary beta gyres. These results agree with Gilet et al. (2009), who show the key role played by the barotropic PV gradient. Vandermeirsch et al. (2003) investigated the trajectory of oceanic eddies in both vertically and horizontally sheared currents. More precisely, they studied the crossing of a baroclinically unstable zonal jet by a vortex in a 2.5 -layer model, showing that strong enough lower-layer anticyclonic vortices can cross the jet by forming a dipole with a cyclonic meander of the upper-layer jet.

The present paper deals with the combined effects of the large-scale horizontal deformation and nonlinearities on the $\beta$-drift motion of surface midlatitude depressions by using a two-layer quasigeostrophic model. As in Oruba et al. (2012), the flow is separated into a largescale basic flow and a perturbation. Section 2 describes the baroclinic model and provides information on the setting of the initial perturbations. In section 3 , the linear and nonlinear evolution of a cyclone embedded in a large-scale pure baroclinic zonal flow without horizontal shear is studied on the $f$ plane and $\beta$ plane. This case allows clarifying the role of the barotropic PV gradient in the absence of deformation. Section 4 describes the case of a spatially meandering jet, similar to the case of Oruba et al. (2012) in a barotropic context, but with a vertical profile making it baroclinically unstable. The basic flow is maintained artificially stationary to reproduce the low-frequency flow of Fig. 1, which does not evolve much during the evolution of the surface cyclones. We first compare the evolution of two surface cyclonic eddies initialized south of the jet in two distinct deformation environments (one leading to a strong elongation of the eddy and the other keeping it more or less isotropic). Then a statistical approach is followed by performing hundreds of simulations with various initial locations for the surface cyclonic eddies on the south side of the jet. Finally, conclusions are given in section 5 .

\section{The numerical framework}

\section{a. The baroclinic model}

The Phillips (1951) quasigeostrophic baroclinic twolayer model is used. The horizontal domain is a twodimensional biperiodic plane $(x, y)$ located in the Northern Hemisphere. The numerical model is pseudospectral, computed on a regular grid, and the temporal scheme is a leapfrog one. The spatial and temporal resolutions of the numerical model are equal to
$\Delta x=\Delta y=62.5 \mathrm{~km}$ and $\Delta t=112 \mathrm{~s}$. The size of the domain is $L_{x}=16000 \mathrm{~km}, L_{y}=8000 \mathrm{~km}$. This baroclinic model consists of the advection of potential vorticity $q$ in both layers:

$$
\begin{aligned}
& q_{u}=\nabla^{2} \psi_{u}+f_{0}+\beta y-\lambda^{-2}\left(\psi_{u}-\psi_{l}\right), \\
& q_{l}=\nabla^{2} \psi_{l}+f_{0}+\beta y+\lambda^{-2}\left(\psi_{u}-\psi_{l}\right),
\end{aligned}
$$

and

$$
\frac{\partial q_{k}}{\partial t}+\mathbf{u}_{k} \cdot \nabla q_{k}=F_{k}
$$

where $k \in\{u, l\}$ denotes the upper or lower layer. The geostrophic wind is denoted as $\mathbf{u}_{k}=\left(u_{k}, v_{k}\right)$ and $\psi_{k}$ is the streamfunction in the layer $k$. The Coriolis parameter $f=f_{0}+\beta y$ linearly depends on $\beta$ and $\nabla^{2}$ is the twodimensional Laplacian operator. The parameter $\lambda$ is the Rossby radius of deformation, which is here equal to $450 \mathrm{~km}$.

The flow is separated into a large-scale background flow, denoted with bars and maintained stationary [through the forcing term $F_{k}$ in Eq. (3) equal to $\overline{\mathbf{u}}_{k} \cdot \nabla \bar{q}_{k}$ ], and a perturbation denoted with primes such that

$$
q_{k}(x, y, t)=\bar{q}_{k}(x, y)+q_{k}^{\prime}(x, y, t) .
$$

Following Eq. (3) the evolution of the perturbation PV can be expressed as

$$
\frac{\partial q_{k}^{\prime}}{\partial t}+\overline{\mathbf{u}}_{k} \cdot \nabla q_{k}^{\prime}+\mathbf{u}_{k}^{\prime} \cdot \nabla \bar{q}_{k}+\mathbf{u}_{k}^{\prime} \cdot \nabla q_{k}^{\prime}=0 .
$$

The purpose of maintaining the basic flow stationary is to reproduce a situation found in some observed midlatitude cyclones such as those shown in Fig. 1. During the evolution of these cyclones, it was possible to separate the flow into a low- and a high-frequency part such that the low-frequency part does not change much and corresponds to a well-established weather regime (Vautard 1990). In other words, the large-scale environment in which the cyclones evolved was found to be quasi stationary in those particular cases. This situation should not be considered as systematic since some strong cyclones may occur during weather regime transitions (Colucci 1985).

\section{b. The initial perturbations}

The model is initialized with cyclonic perturbations in both lower and upper layers such that the axis connecting the centers of the upper-layer and lower-layer disturbances is tilted against the large-scale vertical shear. This configuration of perturbation isolines tilting against the shear is known to be favorable for generation of 
potential energy and for baroclinic interaction (see, e.g., Pedlosky 1987). The initial perturbations are defined in terms of relative vorticity $\left(\zeta_{k}^{\prime}=\nabla^{2} \psi_{k}^{\prime}\right)$ as follows:

$$
\begin{aligned}
& \zeta_{u}^{\prime}=A_{u} \exp \left[-\frac{\left(x-x_{0}+x_{d}\right)^{2}+\left(y-y_{0}+y_{d}\right)^{2}}{r^{\prime 2}}\right], \\
& \zeta_{l}^{\prime}=A_{l} \exp \left[-\frac{\left(x-x_{0}\right)^{2}+\left(y-y_{0}\right)^{2}}{r^{\prime 2}}\right],
\end{aligned}
$$

where $A_{u}\left(A_{l}\right)$ is the vorticity maximum in the upper (lower) layer and $r^{\prime}$ is the characteristic radius of the anomalies (common to both the upper-layer and lowerlayer anomaly). The parameters chosen for the cyclones are such that $\max \left(\zeta_{k}^{\prime}\right)=A_{k}=1.5 \times 10^{-4} \mathrm{~s}^{-1}$ and $\max \left(v_{k}^{\prime}\right)=22 \mathrm{~m} \mathrm{~s}^{-1}$, which leads to $r^{\prime} \approx 463 \mathrm{~km}$. The center of the lower-layer perturbation is located at $\left(x_{0}, y_{0}\right)$. The upper-layer perturbation has the same shape and amplitude as the lower-layer one but is located upstream at the location $\left(x_{0}-x_{d}, y_{0}-y_{d}\right)$. The purpose of choosing such a configuration is to fit with the real cases shown in the bottom panels of Fig. 1 where two finiteamplitude cyclonic anomalies were found to interact with each other with the lower one being located downstream of the upper one. The upper-layer perturbation is such that the upper-layer and lower-layer cyclones will initially have a strong baroclinic interaction. This scenario in which the upper-level PV anomalies reinforce the lower-layer ones and vice versa relies on the interpretation of baroclinic instability in terms of two counterpropagating Rossby waves interacting with each other, first described by Bretherton (1966) in the Eady and Phillips models. This last mechanism has been especially extended to more general zonal flows with a continuous vertical profile in a model based on the conservation of PV by Heifetz et al. (2004) and then used in a linearized primitive equation model by Methven et al. (2005a), the latter results providing robust predictions for the nonlinear evolution of baroclinic structures (Methven et al. 2005b). Such an optimal configuration for potential energy extraction by the disturbances is obtained when the two disturbances are in quadrature phase (Davies and Bishop 1994) and for an axis that is parallel to the background isotherms. To satisfy these two criteria, the maximum of $\zeta_{u}^{\prime}$ is chosen to be upstream of the maximum of $\zeta_{l}^{\prime}$, along the local basic-state streamlines (approximated by the local tangent), at a distance corresponding to that between the maxima of $\zeta_{l}^{\prime}$ and $v_{l}^{\prime}$.

\section{Horizontally uniform zonal flow}

In the present section, the basic flow is composed of a horizontally uniform zonal flow $\left(\bar{u}_{u}=12.5 \mathrm{~m} \mathrm{~s}^{-1}\right.$ and $\left.\bar{u}_{l}=-12.5 \mathrm{~m} \mathrm{~s}^{-1}\right)$. In this setting we will characterize Rossby wave radiation in a relatively simple baroclinic environment. Owing to the existence of PV gradients $\partial_{y} \bar{q}_{u}=\beta+\lambda^{-2}\left(\bar{u}_{u}-\bar{u}_{l}\right)$ and $\partial_{y} \bar{q}_{l}=\beta-\lambda^{-2}\left(\bar{u}_{u}-\bar{u}_{l}\right)$, Rossby waves will be emitted in each layer by both cyclones. As explained in Gilet et al. (2009), we expect a northward motion of the lower-layer cyclone only in the nonlinear simulation for which the barotropic PV gradient [here equal to $\partial_{y}\left(\bar{q}_{u}+\bar{q}_{l}\right) / 2=\beta$ ] is not zero. This hypothesis is tested here by inspecting the spatial evolution of the perturbation flow. The initial lower-layer (upperlayer) cyclones are located at $x_{0}=0, y_{0}=0\left(x_{0}-x_{d}=\right.$ $-500 \mathrm{~km}, y_{0}-y_{d}=0$ ) (see Fig. 2a). The initial perturbation PV field associated with both cyclones comprises a strong positive anomaly in each layer and slightly negative anomalies to the east in the upper layer and to the west in the lower one (Fig. 2b). The value of $\beta$ chosen here is either zero or $4.8 \times 10^{-11} \mathrm{~m}^{-1} \mathrm{~s}^{-1}$ - that is to say 3 times the common value - to reproduce the additional relative vorticity gradient created by the presence of a meridionally confined jet as in the next section. Linear and nonlinear simulations are then performed.

The PV fields at $t=15 \mathrm{~h}$ for the linear and nonlinear simulations with $\beta=0$ and with nonzero $\beta$ are shown in Fig. 3. In the linear simulation with $\beta=0$ (Fig. 3a), an anticyclone develops to the west (east) of the lower (upper)-layer cyclone. This is due to the meridional advection of the basic-state PV by the initial cyclones. At the upper layer, as the basic-state PV gradient points northward $\left[\partial_{y} \bar{q}_{u}=\lambda^{-2}\left(\bar{u}_{u}-\bar{u}_{l}\right)>0\right]$, an anticyclone is formed in the region of poleward velocity, that is, to the east of the cyclone. On the contrary, at the lower layer, the southward-oriented basic-state $\mathrm{PV}$ gradient $\left[\partial_{y} \overline{q_{l}}=\right.$ $\left.-\lambda^{-2}\left(\bar{u}_{u}-\bar{u}_{l}\right)<0\right]$ leads to the development of an anticyclone to the west of the cyclone. As the upper-layer and lower-layer potential vorticity gradients are equal in absolute value, the upper-layer and lower-layer anticyclones have the same amplitude. This scenario is typical of energy dispersion by Rossby wave radiation (e.g., Flierl 1977) and would lead to the development of other cyclonic and anticyclonic PV anomalies farther in the zonal direction for later times.

Adding $\beta$ (Fig. 3b) leads to a more positive upperlayer PV gradient $\partial_{y} \bar{q}_{u}=\beta+\lambda^{-2}\left(\bar{u}_{u}-\bar{u}_{l}\right)$ and more energy dispersion to the east, in agreement with Simmons and Hoskins (1979). In the upper layer, the anticyclone is therefore stronger and the cyclone weaker than in the $\beta=0$ case. The case with nonzero $\beta$ induces a less negative PV gradient $\partial_{y} \overline{q_{l}}=\beta-\lambda^{-2}\left(\bar{u}_{u}-\bar{u}_{l}\right)$ in the lower layer, leading to a weaker anticyclone to the west of the cyclone than in the $\beta=0$ case.

In the nonlinear simulation with $\beta=0$ (Fig. 3c), the upper-layer and lower-layer anticyclones have the same 
a) Initial relative vorticity

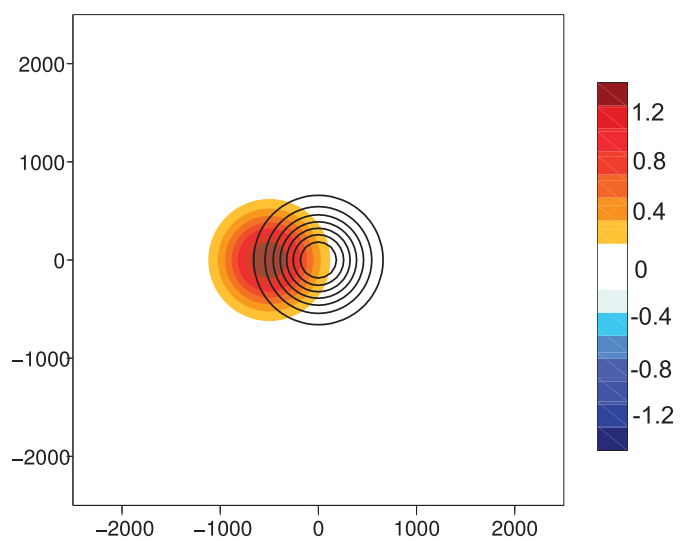

b) Initial potential vorticity

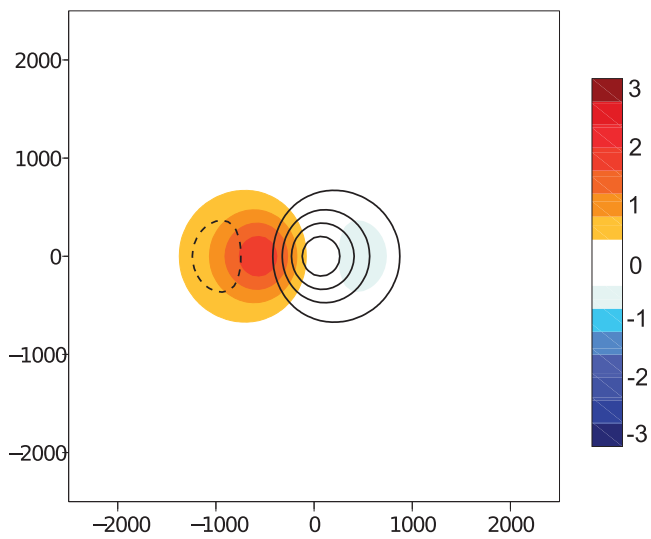

FIG. 2. Initial perturbation (a) relative and (b) potential vorticity fields $\left(\mathrm{s}^{-1}\right)$ in the lower (black contours) and upper (color shading) layers. In (a) positive values are represented by solid contours from $1.8 \times 10^{-5} \mathrm{~s}^{-1}$ every $2 \times$ $10^{-5} \mathrm{~s}^{-1}$ and negative values are represented by dashed contours from $-1.8 \times 10^{-5} \mathrm{~s}^{-1}$ every $2 \times 10^{-5} \mathrm{~s}^{-1}$ (color shading; $10^{-4} \mathrm{~s}^{-1}$ ). In (b) positive values are represented by solid contours between $4 \times 10^{-5}$ and $28 \times 10^{-5} \mathrm{~s}^{-1}$ every $4 \times 10^{-5} \mathrm{~s}^{-1}$ and negative values are represented by dashed contours between $-28 \times 10^{-5}$ and $-4 \times 10^{-5} \mathrm{~s}^{-1}$ every $4 \times 10^{-5} \mathrm{~s}^{-1}$. Latitude and longitude are in kilometers.

amplitude, as the upper-layer and lower-layer PV gradients are equal in absolute value. Since both anticyclones are weaker than the corresponding cyclones, the former are stretched by the latter and tend to turn around them. As for the linear simulations, adding $\beta$ leads to a stronger upper-layer anticyclone and weaker lower-layer anticyclone than in the $\beta=0$ case (see Figs. $3 \mathrm{c}, \mathrm{d})$. When the upper-layer anticyclone is sufficiently strong, it forms a dipolar anomaly with the upper-layer cyclone that is mainly zonally oriented. By the principle of PV inversion, the upper PV dipole thus induces a meridional velocity at the center of the lower-layer cyclone, which explains its poleward drift. The lower-layer cyclone in the case with $\beta$ (Fig. 3d) has, indeed, a rapid poleward motion and reaches the latitude $y=400 \mathrm{~km}$ at $t=15 \mathrm{~h}$, whereas it did not move meridionally in the linear case (Fig. 3b). For the nonlinear $\beta=0$ case (Fig. 3c), there is no net displacement toward the pole because the lower-layer PV dipole, characterized by an anticyclone to the west of the cyclone, counterbalances the drift attributed to the upper-level PV dipole.

In conclusion, the barotropic PV gradient leads to an asymmetry between the upper and lower layers with more efficient energy dispersion through Rossby wave radiation in the upper layer than in the lower layer. As a result, it is responsible for the formation of an upperlayer anticyclone that is stronger than the lower-layer anticyclone, which also develops. The vortex dipole formed by the upper-layer cyclone and the upperlayer anticyclone is then responsible for the poleward drift of the lower-layer cyclone through a nonlinear effect. Our scenario gives a physical interpretation of the results of Gilet et al. (2009). Hereafter, the influence of the horizontal deformation on this nonlinear mechanism is investigated in a horizontally inhomogeneous flow.

\section{Spatially meandering jet}

\section{a. Description of the basic flow}

To reproduce the large-scale flows of Fig. 1, a spatially nonuniform jet that is meandering is introduced; its streamfunction is defined by

$$
\overline{\psi_{k}}=-\frac{u_{0}^{k} \sqrt{\pi}}{2 \alpha} \operatorname{erf}\{\alpha[y-\epsilon \sin (K x-\pi / 4)]\},
$$

where

$$
\operatorname{erf}\{l\}=\frac{2}{\sqrt{\pi}} \int_{0}^{l} \exp \left(-s^{2}\right) d s .
$$

The horizontal geostrophic wind field can be written as

$$
\left\{\begin{array}{l}
\bar{u}_{k}=u_{0}^{k} \exp \left(-\{\alpha[y-\epsilon \sin (K x-\pi / 4)]\}^{2}\right) \\
\bar{v}_{k}=u_{0}^{k} \epsilon K \cos (K x-\pi / 4) \exp \left(-\{\alpha[y-\epsilon \sin (K x-\pi / 4)]\}^{2}\right) .
\end{array}\right.
$$


a) Linear case with $\beta=0$

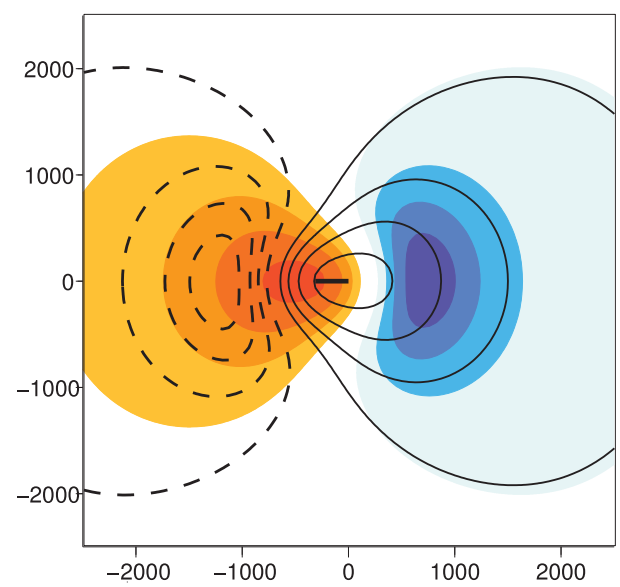

c) Nonlinear case with $\beta=0$

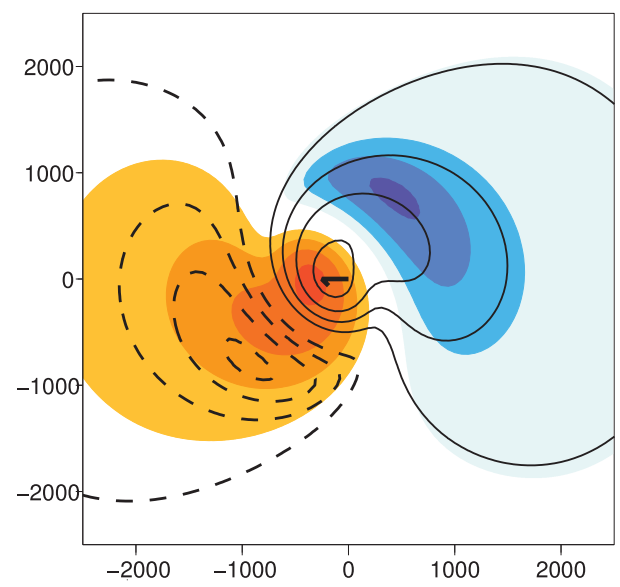

b) Linear case with $\beta \neq 0$

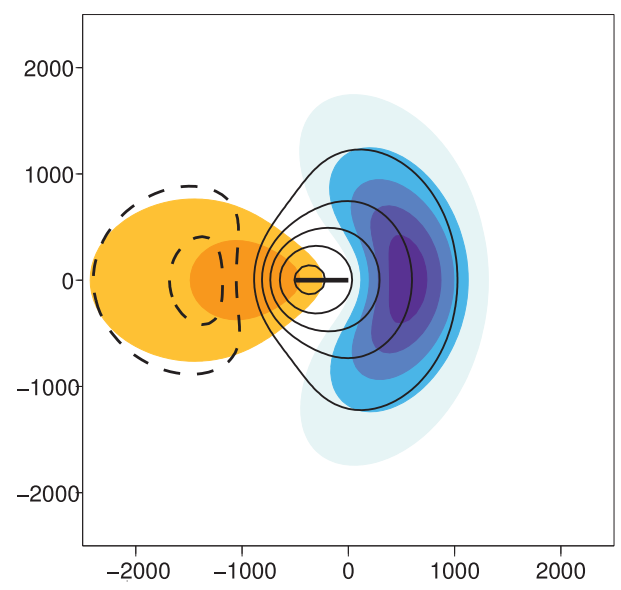

d) Nonlinear case with $\beta \neq 0$

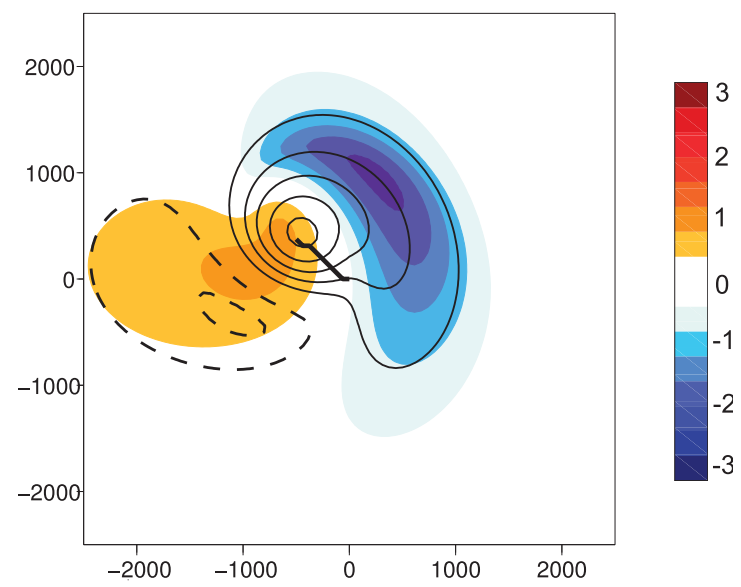

FIG. 3. Potential vorticity fields $\left(\mathrm{s}^{-1}\right)$ at $t=15 \mathrm{~h}$ for (a) linear simulation with $\beta=0$, (b) linear simulation with $\beta=4.8 \times 10^{-11} \mathrm{~m}^{-1} \mathrm{~s}^{-1}$, (c) nonlinear simulation with $\beta=0$, and (d) nonlinear simulation with $\beta=4.8 \times$ $10^{-11} \mathrm{~m}^{-1} \mathrm{~s}^{-1}$ : legend as in Fig. $2 \mathrm{~b}$. The thick line segment represents the trajectory of the lower-layer cyclone (defined as the successive positions of the maximum of perturbation relative vorticity in the lower layer).

The jet maximum (or jet core) is defined as the maximum zonal wind speed whose isoline satisfies $y=$ $\epsilon \sin (K x-\pi / 4)$. The different parameters of the jet are defined in Table 1.

Figure 4a shows the background zonal wind profile as a function of latitude, defined in Eq. (8), for $x=$ $1000 \mathrm{~km}$. The zonal wind is twice as large in the upper layer as in the lower layer. Figure 5 shows the streamlines of the lower-layer meandering jet for the meander parameters $2 \pi K^{-1}=8000 \mathrm{~km}$ and $4 \epsilon=2500 \mathrm{~km}$. Figure $4 \mathrm{~b}$ shows the meridional gradient of the background $\mathrm{PV}$ as a function of latitude for $x=1000 \mathrm{~km}$. It is not spatially uniform, but points mainly poleward in the upper layer, with a maximum value equal to $2 \times 10^{-10} \mathrm{~m}^{-1} \mathrm{~s}^{-1}$, and it points mainly equatorward in the lower layer (except on the meridional edge of the domain), with a minimum value equal to $-0.7 \times 10^{-10} \mathrm{~m}^{-1} \mathrm{~s}^{-1}$. This configuration with opposite PV gradients in both layers is favorable for baroclinic instability (see, e.g., Pedlosky 1987; Davies and Bishop 1994). However, the vertically averaged PV

TABLE 1. Model parameters.

\begin{tabular}{cccccccccc}
\hline \hline$f_{0}\left(\mathrm{~s}^{-1}\right)$ & $\beta\left(\mathrm{m}^{-1} \mathrm{~s}^{-1}\right)$ & $\lambda(\mathrm{m})$ & $u_{0}^{u}\left(\mathrm{~m} \mathrm{~s}^{-1}\right)$ & $u_{0}^{l}\left(\mathrm{~m} \mathrm{~s}^{-1}\right)$ & $L_{x}(\mathrm{~km})$ & $L_{y}(\mathrm{~km})$ & $K\left(\mathrm{~m}^{-1}\right)$ & $\epsilon(\mathrm{km})$ & $\alpha\left(\mathrm{m}^{-1}\right)$ \\
\hline $10^{-4}$ & $1.6 \times 10^{-11}$ & $4.5 \times 10^{5}$ & 50 & 25 & 16000 & 8000 & $\frac{4 \pi}{L_{x}} \simeq 8 \times 10^{-7}$ & $\frac{L_{y}}{4 \pi} \simeq 640$ & $\frac{2 \pi}{L_{y}} \simeq 8 \times 10^{-7}$ \\
\hline
\end{tabular}




\section{a) Zonal speed}

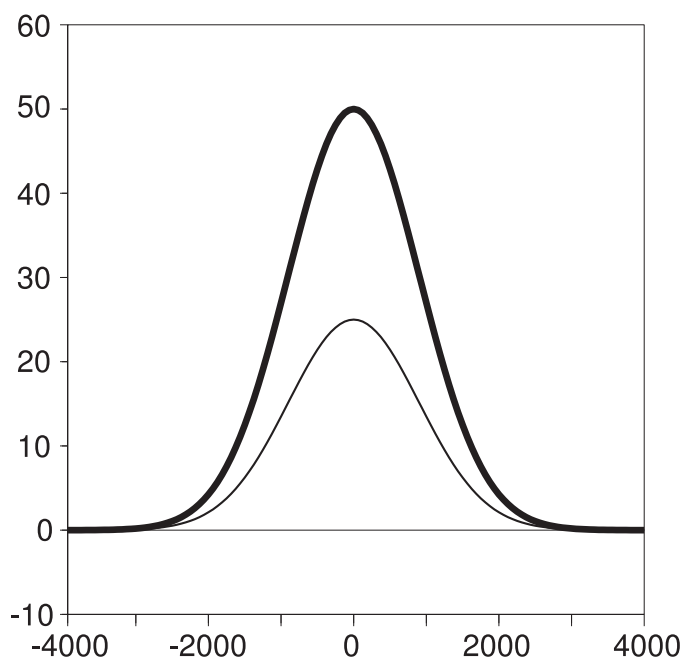

\section{b) Meridional gradient of PV}

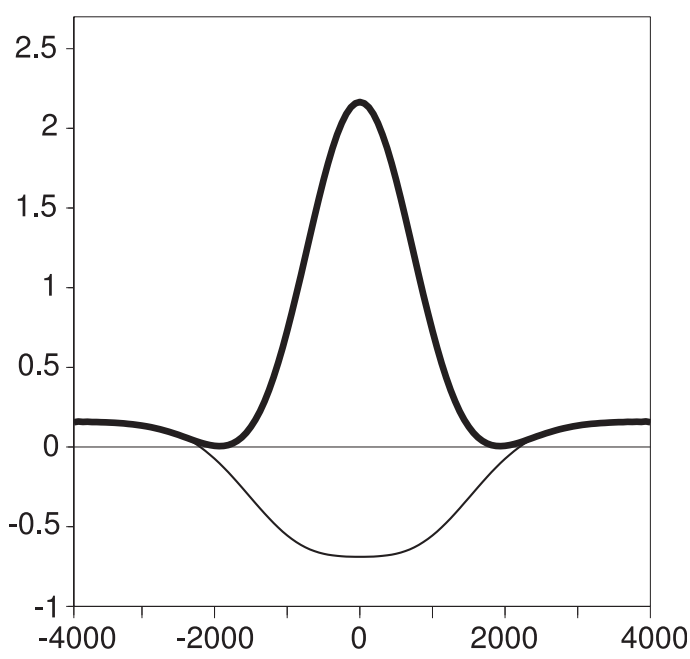

FIG. 4. (a) Zonal speed $\left(\mathrm{m} \mathrm{s}^{-1}\right)$ associated with the basic state (for $x=1000 \mathrm{~km}$ ) as a function of latitude $(y, \mathrm{~km})$. (b) Meridional gradient of PV $\left(10^{-10} \mathrm{~m}^{-1} \mathrm{~s}^{-1}\right)$ of the basic state as a function of latitude: upper layer (thick) and lower layer (thin).

gradient is strongly positive because of $\beta$ and the relative vorticity gradient due to the presence of a meridionally confined jet. This quantity is the key parameter explaining the poleward motion of the surface cyclones, as explained in the previous section. Such a flow is chosen because the PV isolines and the streamlines are almost parallel (see Fig. 5). From Eq. (5) it can be deduced that a cyclone in the linear case will move along the basicstate streamlines. This would not be the case if the streamlines were not parallel to the PV isolines as the maximum of PV would also develop along the PV isolines in addition to being advected by the flow (along the streamlines). In consequence, our setting precludes linear effects to displace cyclones across the basic-state streamlines and the jet axis.

The horizontal deformation tensor associated with the basic-state velocity field $\overline{\mathbf{u}}=(\bar{u}(x, y), \bar{v}(x, y))$ can be described by its deformation magnitude $\bar{D}$ :

$$
\bar{D}=\sqrt{\left(\frac{\partial \bar{v}}{\partial x}+\frac{\partial \bar{u}}{\partial y}\right)^{2}+\left(\frac{\partial \bar{u}}{\partial x}-\frac{\partial \bar{v}}{\partial y}\right)^{2}},
$$

and its relative vorticity $\bar{\zeta}$ :

$$
\bar{\zeta}=\frac{\partial \bar{v}}{\partial x}-\frac{\partial \bar{u}}{\partial y} .
$$

The quantity $\bar{D}$ characterizes the straining effects that tend to elongate any perturbation. On the contrary, $\bar{\zeta}$ causes any perturbation to rotate cyclonically or anticyclonically, depending on the sign of $\bar{\zeta}$. Perturbations do not necessarily elongate in regions of stronger $\bar{D}$ but in regions where the following quantity is positive (Rivière et al. 2003):

$$
\Delta=\bar{D}^{2}-\bar{\zeta}^{2}
$$

When $\Delta>0$, the straining effects dominate the rotation and the perturbation will be stretched. When $\Delta<0$, the

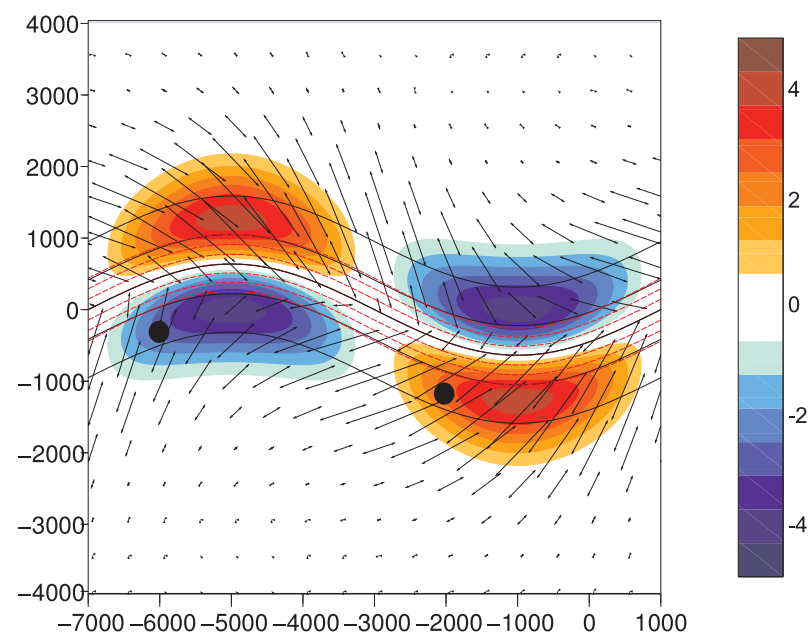

FIG. 5. Effective deformation $\Delta$ field of the lower-layer basic state (color shading; $10^{-10} \mathrm{~s}^{-2}$ ) and dilatation axis (arrows). Streamlines in the lower layer (black contours between $-3 \times 10^{7}$ and $3 \times$ $10^{7} \mathrm{~m}^{2} \mathrm{~s}^{-1}$ every $10^{7} \mathrm{~m}^{2} \mathrm{~s}^{-1}$ ) and jet center (thick line); iso- $\overline{q_{l}}$ lines (red dashed contours between $-3 \times 10^{-5}$ and $4 \times 10^{-5} \mathrm{~s}^{-1}$ every $10^{-5} \mathrm{~s}^{-1}$ ); initial locations (black patches) of the two lower-layer cyclones studied in section 4. Latitude and longitude are in kilometers. 
rotation effects dominate and the perturbation will not be elongated very much. This criterion was applied in real cases by Rivière and Joly (2006a), who called it effective deformation. The $\Delta$ quantity is, in fact, the Okubo-Weiss criterion (Okubo 1970; Weiss 1981) applied to the basic state (or to the low-frequency flow in real cases). We note that Lapeyre et al. (1999) have proposed a modified version of the Okubo-Weiss criterion to take into account its non-Galilean invariance (and the rotation of the strain axes). In the case of slightly meandering jets, the Okubo-Weiss criterion is adequate while it is less adequate for flows with strong curvature (Lapeyre et al. 1999; Rivière et al. 2003). Note also that Cohen and Schultz (2005) have introduced this quantity to diagnose fronts or airstream boundaries in the troposphere.

Figure 5 shows the effective deformation field $\Delta$ for the lower layer. It exhibits positive values on the northern (southern) side of the ridges (troughs) and reaches values of the order of $4.7 \times 10^{-10} \mathrm{~s}^{-2}$ in the lower layer and $1.9 \times$ $10^{-9} \mathrm{~s}^{-2}$ in the upper layer, similar to the values reached in Fig. 1. Note finally that the alternating cyclonic and anticyclonic curvatures reproduce quite well the lowfrequency effective deformation field of Fig. 1 and the related saddle points without introducing any jet entrance or exit regions.

\section{b. Study of two generic cases}

\section{1) DESCRIPTION OF THE TIME EVOLUTION}

Figure 6 shows the temporal evolution of the perturbation relative vorticity for two simulations initialized with a lower-layer cyclone located at distance $d=750 \mathrm{~km}$ to the south of the jet core, upstream of regions of negative and positive $\Delta$ (see black patches in Fig. 5). The position of the perturbations upstream of negative delta is $(x, y)=(-6000,-430) \mathrm{km}$ for the lower layer and $(x, y) \approx(-6470,-630) \mathrm{km}$ for the upper layer, while it is respectively $(x, y)=(-2000,-1330) \mathrm{km}$ and $(x, y) \approx$ $(-2470,-1120) \mathrm{km}$ for the perturbations upstream of positive delta. The axis connecting the centers of upper and lower disturbances is initially parallel to the background streamlines (Figs. 6a,b) for optimizing baroclinic interaction as explained in section $2 \mathrm{~b}$. Figure 7 shows the corresponding perturbation PV field at $t=15 \mathrm{~h}$ and $t=$ $35 \mathrm{~h}$. Note that the setting with disturbances initially located upstream of the region of positive $\Delta$ (Figs. 6b,d,f) is the most realistic one since it corresponds to the real cases shown in Fig. 1.

The black line in Figs. 6c-f (and in Figs. 7a-d for PV) is the trajectory of the lower-layer cyclone by following the relative vorticity maximum. It crosses the large-scale jet (that is to say the basic-state streamlines) from its equatorward side to its poleward side in both cases. We have checked that linear simulations do not exhibit such a behavior and that the crossing of the basic-state isolines is a purely nonlinear effect.

At $t=15 \mathrm{~h}$, the lower-layer cyclone initialized in the area where $\Delta<0$ is slightly deformed (Fig. 6c) whereas its counterpart, initialized in the area where $\Delta>0$, has a much more elongated shape (consistent with the definition of $\Delta$ ) and is tilted in a southwest-northeast direction (Fig. 6d). This elongation direction is more meridionally oriented than that of the local dilatation axis of the background flow, which is southwestnortheast oriented in this region (Fig. 5) because of the additional effect of nonlinear anticlockwise self-rotation of the cyclone, as described by Gilet et al. (2009) and Oruba et al. (2012).

As can be observed in Fig. 4b, the upper-layer largescale meridional PV gradient is poleward oriented and greater (in absolute value) than the equatorwardoriented lower-layer one. According to the results of section 3, strong energy dispersion by Rossby wave radiation occurs to the east of the upper-layer cyclone whereas, in the lower layer, energy dispersion is weaker and occurs to the west of the cyclone. As a result, we observe a strong anticyclone to the east of the cyclone in the upper layer (see the blue shadings in Figs. 7a-d) and a comparatively weaker anticyclone to the west of the cyclone in the lower layer (see the dashed contours in Figs. 7a-d). Note that the PV and relative vorticity anomalies slightly differ from each other. There are two negative relative vorticity anomalies in the lower layer (Figs. 6c-f) to the west and east of the cyclone. The latter anomaly probably results from baroclinic interaction through the advection of the lower large-scale PV by the strong upper-layer anticyclone.

Both intensity and location of the upper-layer anticyclone depend on the initial location of the cyclone. Indeed, at $t=15 \mathrm{~h}$ the upper-layer anticyclone is stronger in amplitude for the most deformed cyclone than for the less deformed case (cf. Figs. 6c and 6d). Moreover, at both $t=15 \mathrm{~h}$ and $t=35 \mathrm{~h}$, in the former case, the anticyclone is more coherent and more to the east of the cyclone than in the latter case where it tends to sprawl and to curl around the cyclone (cf. Figs. 6e and 6f). In addition, at $t=35 \mathrm{~h}$ the deformed cyclone has already crossed the jet axis (its crossing time is approximately at $25.5 \mathrm{~h}$ ), whereas the less deformed one has not (its crossing time is approximately at $40.5 \mathrm{~h})$. On the contrary, the zonal displacement is almost the same $(\sim 3000 \mathrm{~km})$.

We conclude that deformation effects modulate the effect of the barotropic PV gradient presented in section 3 by reinforcing energy dispersion. Indeed, the most deformed lower-layer cyclone has a stronger upper-layer 
a) Relative vorticity at $\mathrm{t}=0 \mathrm{~h}, \Delta<0$

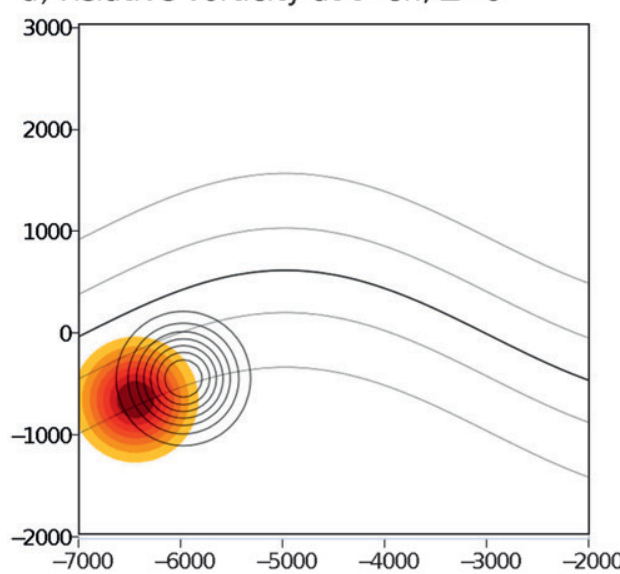

c) Relative vorticity at $\mathrm{t}=15 \mathrm{~h}, \Delta<0$

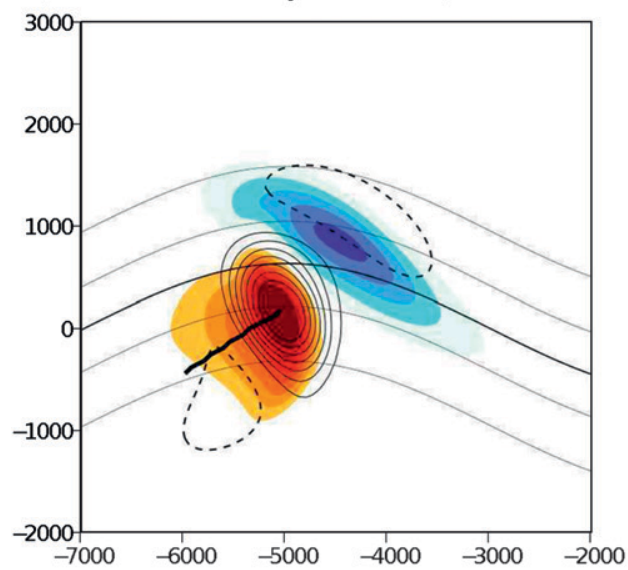

e) Relative vorticity at $\mathrm{t}=35 \mathrm{~h}, \Delta<0$

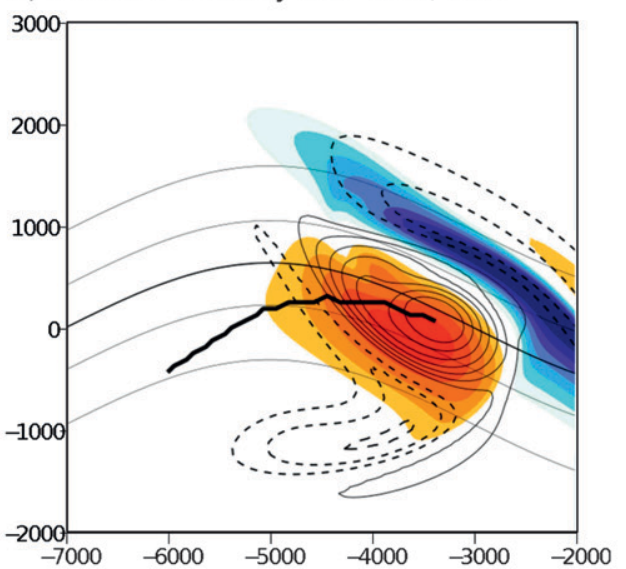

b) Relative vorticity at $\mathrm{t}=0 \mathrm{~h}, \Delta>0$

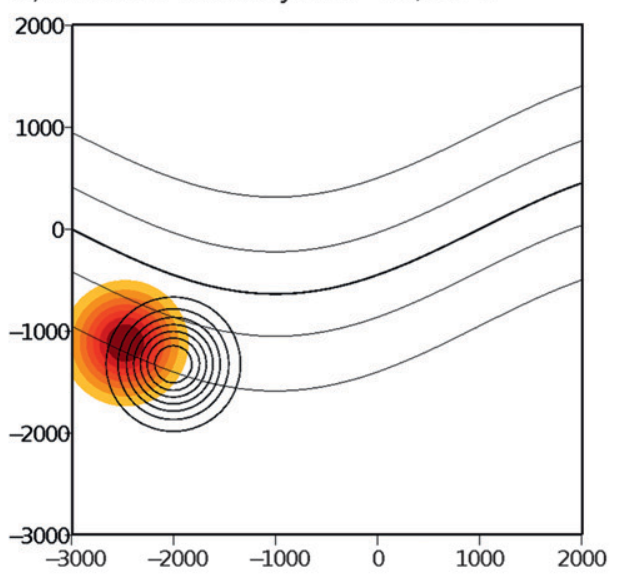

d) Relative vorticity at $\mathrm{t}=15 \mathrm{~h}, \Delta>0$

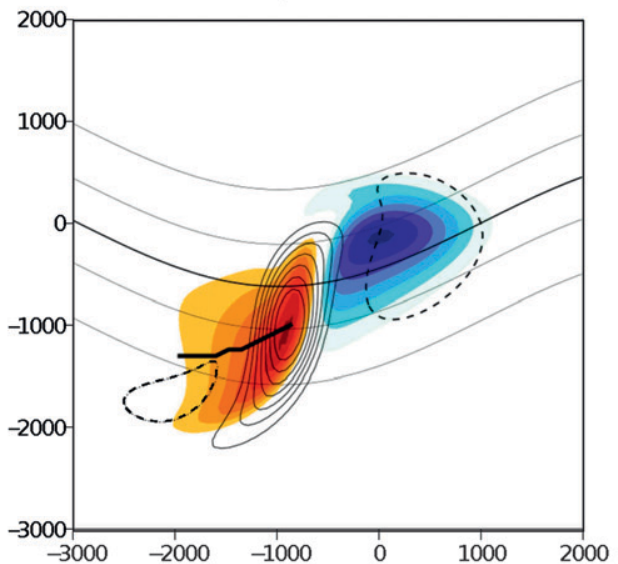

f) Relative vorticity at $\mathrm{t}=35 \mathrm{~h}, \Delta>0$

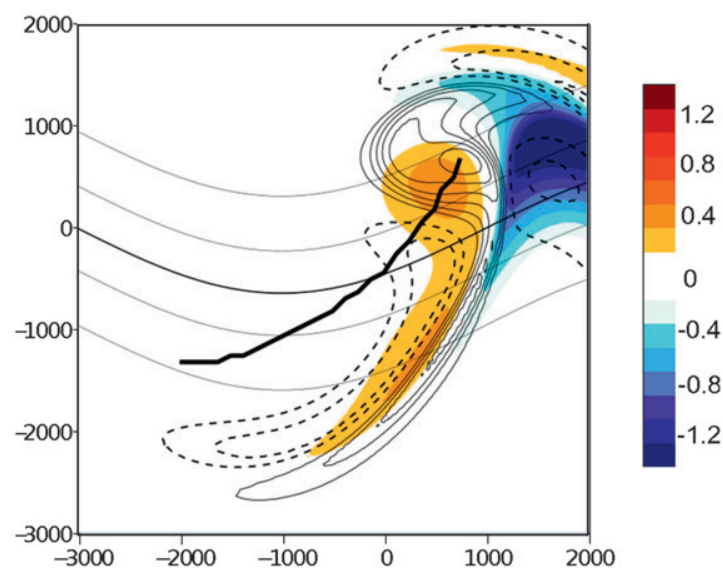

FIG. 6. Time evolution of disturbances initially located in (left) negative and (right) positive effective deformation regions. Relative vorticity field in the lower layer (black contours) at (a),(b) $t=0$, (c),(d) $t=15$, and (e),(f) $t=35 \mathrm{~h}$. Positive values are represented by solid contours from $2 \times 10^{-5} \mathrm{~s}^{-1}$ every $1.8 \times 10^{-5} \mathrm{~s}^{-1}$; negative values by dashed contours from $-2 \times 10^{-5} \mathrm{~s}^{-1}$ every $1.8 \times 10^{-5} \mathrm{~s}^{-1}$. Relative vorticity field in the upper layer (color shading; $10^{-4} \mathrm{~s}^{-1}$ ). The thick line in (c)-(f) indicates the trajectory of the lower-layer cyclone. Streamlines in the lower layer (black contours) and the jet center (thick line). Latitude and longitude are in kilometers. 

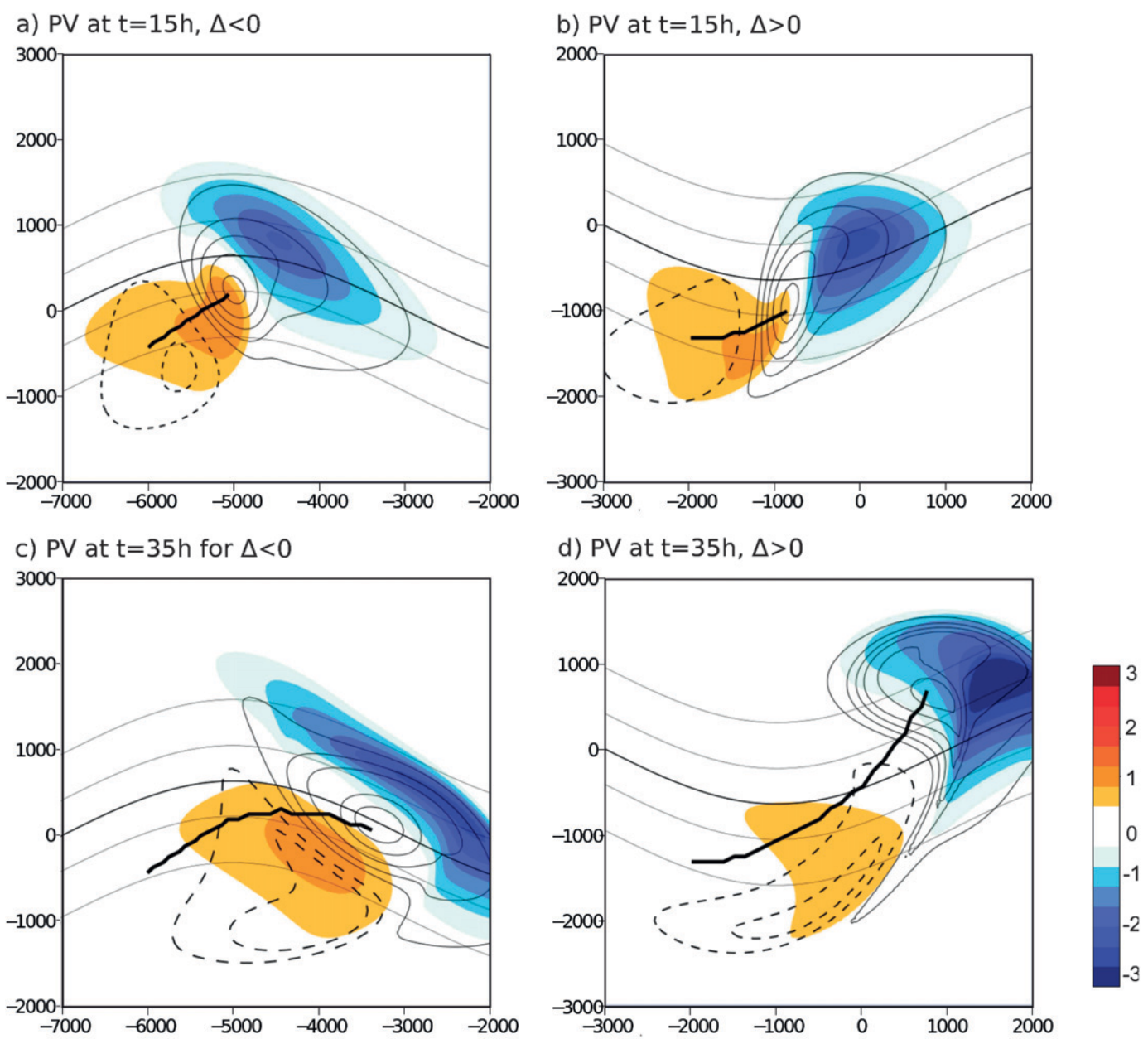

FIG. 7. As in Fig. 6, but for the PV field. Positive values are represented by solid contours every $4 \times 10^{-5} \mathrm{~s}^{-1}$; negative values by dashed contours every $4 \times 10^{-5} \mathrm{~s}^{-1}$ (color shading; $10^{-4} \mathrm{~s}^{-1}$ ).

anticyclone, which remains to the northeast of the cyclone. As the lower-layer cyclone motion depends in part on the circulation induced by the upper-layer anticyclone, the most deformed cyclone crosses the jet axis faster than the slightly deformed one, whose upper-layer anticyclone is weaker and tends to sprawl around it. Thus, when the large-scale barotropic PV gradient or deformation is weak, the development of the upperlayer anticyclone is slower, which leads to a smaller cross-jet displacement of the cyclone [as also discussed in a barotropic context by Oruba et al. (2012)]. The purpose of the next section is to quantify and further explore the role of the deformation in energy dispersion by Rossby wave radiation and the cross-jet motion.

\section{2) ANALYSIS}

The time evolution of the maximum of the lower-layer and upper-layer perturbation relative vorticity (Figs. 8a,b, respectively) and that of the minimum (in absolute value) of the upper-layer perturbation relative vorticity (Fig. 8c) are investigated. The dashed line corresponds to the $\Delta<0$ regions and the solid line to the $\Delta>0$ regions.

The amplitude of the lower-layer cyclone (in terms of maximum of relative vorticity) remains almost constant in time in both cases (Fig. 8a). On the contrary, the intensity of the upper-layer cyclone decreases with time after a few hours (Fig. 8b). At the same time, the amplitude of the upper-layer anticyclone increases (Fig. 8c). This is consistent with the strong energy dispersion in the upper layer, as described in the previous section. The fact that the amplitude of the lower-layer cyclone does not decrease can be attributed to two reasons. First, the basic-state PV gradient is small in the lower layer (Fig. 4b), which causes a weak dispersion of energy. Moreover, the lower-layer cyclone is strengthened by the advection of the lower-layer basic-state PV induced by both the upperlayer cyclone located to the west and the upper-layer 
a) Intensity of the lower cyclone

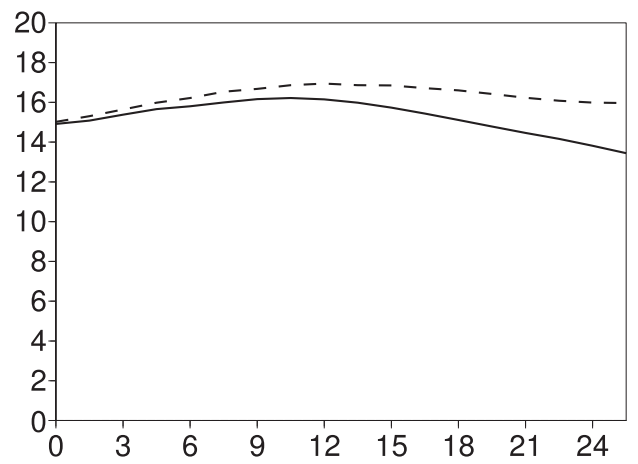

c) Intensity of the upper anticyclone

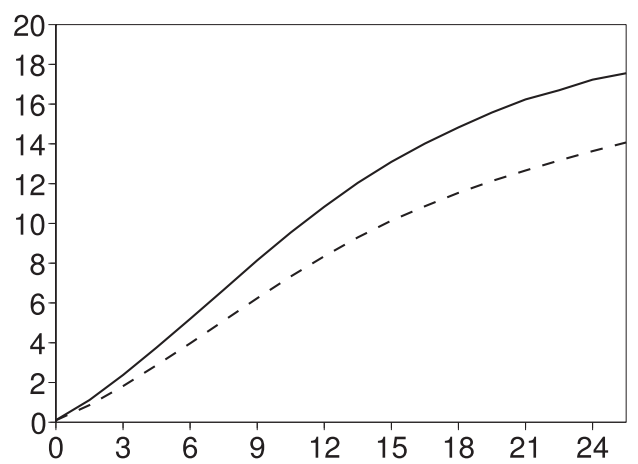

b) Intensity of the upper cyclone

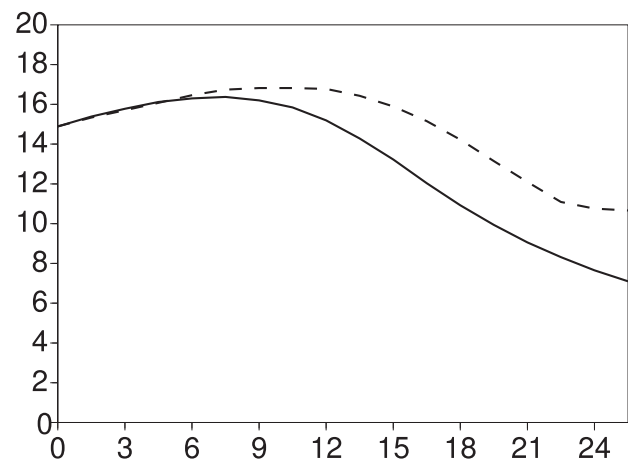

d) Aspect ratio

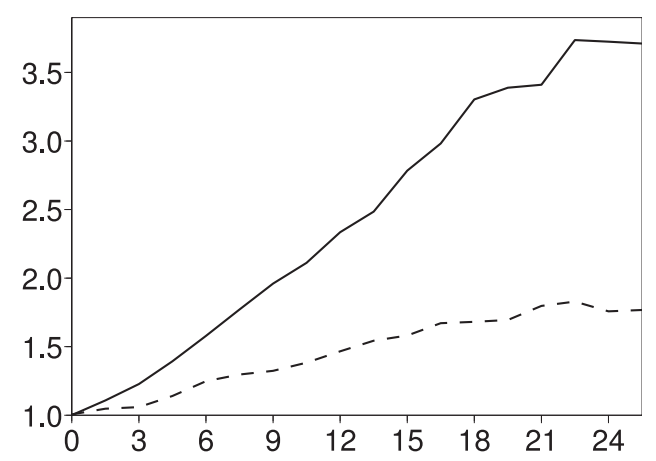

FIG. 8. Evolution with time (h) of the maximum of the (a) lower-layer and (b) upper-layer relative vorticity, (c) of the minimum (absolute value) of the upper-layer relative vorticity $\left(10^{-5} \mathrm{~s}^{-1}\right)$, and (d) of the aspect ratio of the lowerlayer cyclone for disturbances initially located in negative (dashed) and positive (solid) effective deformation regions.

anticyclone located to the east. Since the strengths of the upper-layer cyclone and upper-layer anticyclone respectively decrease and increase with time, the advection induced by both eddies in the lower layer is almost constant with time.

Let us now examine the effect of the deformation on the shape of the lower-layer cyclone and on its cross-jet speed. At first order, the lower-layer cyclone is approximated as an ellipse, and its deformation is quantified by the aspect ratio of the ellipse, defined as the ratio between its major and minor radii. Figure $8 \mathrm{~d}$ shows the time evolution of the aspect ratio of the lower-layer cyclone. During the whole simulation, the lower-layer cyclone initialized in the area where $\Delta<0$ is less deformed than that initialized in the area where $\Delta>0$, as observed in Fig. 6. The basic-state effective deformation $\Delta$ is, indeed, the parameter that governs the deformation of the disturbances (Kida 1981). This tendency is also valid for the upper-layer cyclones (cf. Figs. 6c and 6d). The deformation has different consequences: the deformed upper-layer cyclone weakens more rapidly (Fig. 8b) and the upper-layer anticyclone grows more rapidly (Fig. 8c) for the most deformed cyclone than for the less deformed one. This can be interpreted following the results of Oruba et al. (2012), who showed that, in a barotropic context, dispersion of energy through Rossby wave radiation is more important if the cyclone is stretched in a direction near to that of the basic-state PV gradient, which is the case here (Fig. 6d).

The cross-jet displacement is now analyzed for the two same cases. The lower-layer cyclone cross-jet speed is estimated with the help of the velocity field $\mathbf{u}_{l}^{\prime}(x, y, t)$ taken in the lower-layer cyclone center. More precisely, the lower-layer cyclone motion in the direction of the jet core is estimated by the perturbation velocity component orthogonal to the local basic-state streamline:

$$
v_{l, \perp}^{\prime}(t)=\left(\frac{-\mathbf{u}_{l}^{\prime} \cdot \nabla \bar{\psi}_{u}}{\left\|\nabla \bar{\psi}_{u}\right\|}\right)_{\mathbf{x}=\mathbf{x}_{\text {traj }}}
$$

where $\mathbf{x}_{\text {traj }}$ represents the successive positions of the maximum perturbation relative vorticity in the lower layer.

Figure 9a shows the time evolution of $v_{l, \perp}^{\prime}$. After a transient phase, the less deformed lower-layer cyclone 


\section{a) Cross-jet speed}

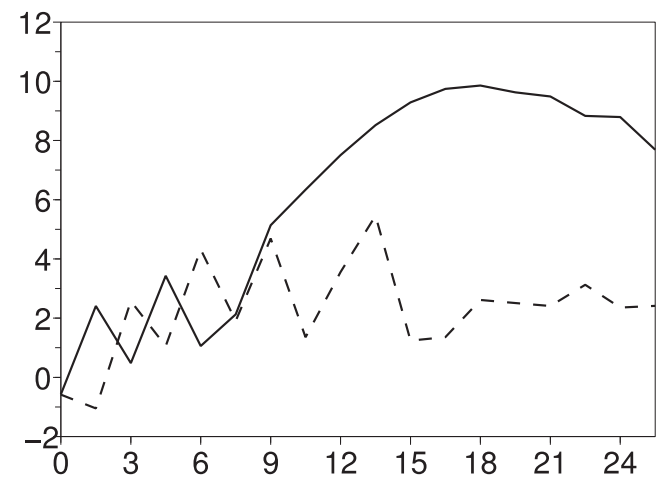

c) Cross-jet speed due to the upper layer

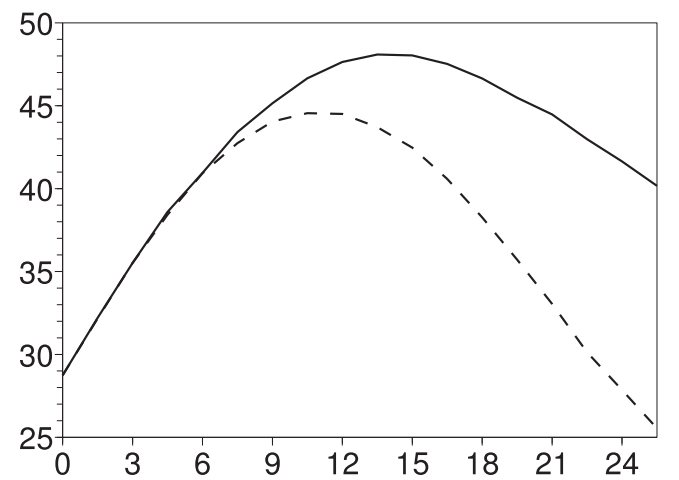

e) Cross-jet speed modulus due to the upper anticyclone

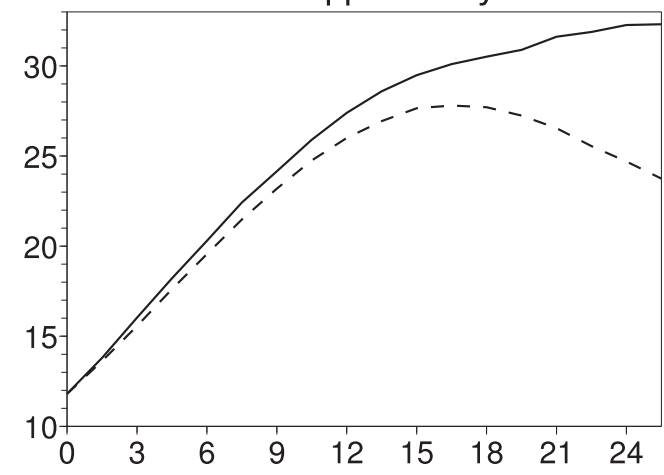

b) Cross-jet speed due to the lower layer

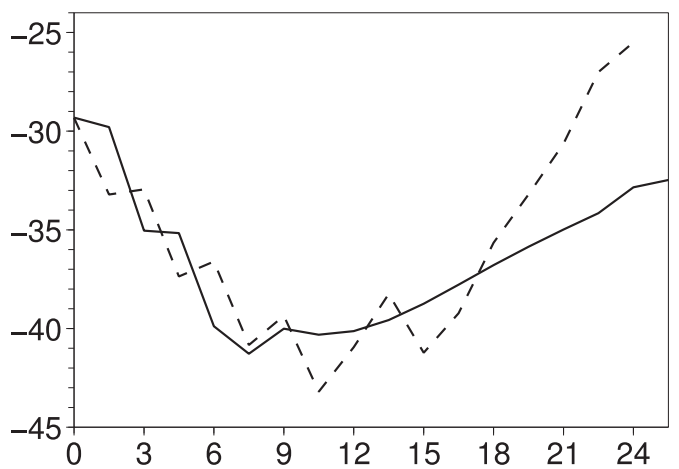

d) Separate effects of upper-layer anomalies

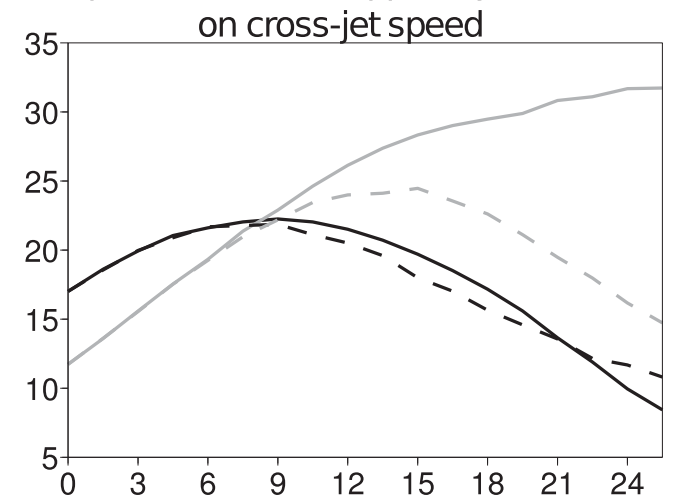

f) Cross-jet speed inclination

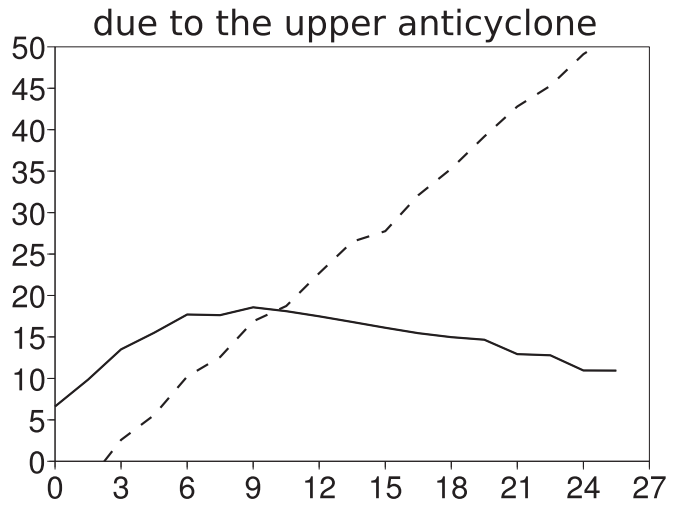

FIG. 9. Evolution with time (h) of (a) $v_{l, \perp}^{\prime}$, (b) $v_{l l, \perp}^{\prime}$, (c) $v_{l u, \perp}^{\prime}$, (d) $v_{l u, \perp}^{\prime \text { ACup }}$ (gray) and $v_{l u, \perp}^{\prime \text { Cup }}$ (black), (e) $\| \mathbf{v}_{l u}^{\prime \text { ACup } \|}\left(\mathrm{m} \mathrm{s}^{-1}\right.$ ) and (f) $\vartheta\left(^{\circ}\right)$. For disturbances initially located in negative (dashed) and positive (solid) effective deformation regions.

has a slower cross-jet speed. Note that $v_{l, \perp}^{\prime}(t=0)=0$ because the initial lower-layer cyclone is defined as a (positive) relative vorticity perturbation, which cannot self-advect. Initializing in terms of PV perturbation would lead to a nonzero initial velocity $v_{l, \perp}^{\prime}$, but the difference between the deformed and nondeformed cyclones would remain qualitatively the same (not shown).

To understand what makes the difference between both cases, we separate the lower-layer perturbation velocity field $\mathbf{u}_{l}^{\prime}$ into two parts: one, denoted $\mathbf{u}_{l l}^{\prime}$, induced by the PV perturbation in the lower layer, and another one, denoted $\mathbf{u}_{l u}^{\prime}$, induced by the PV perturbation in the upper layer. This can be done by the principle of PV inversion: $\mathbf{u}_{l l}^{\prime}\left(\mathbf{u}_{l u}^{\prime}\right)$ is calculated by zeroing $q_{u}^{\prime}\left(q_{l}^{\prime}\right)$ and inverting the PV equations (1) and (2). We note $v_{l l, \perp}^{\prime}$ and $v_{l u, \perp}^{\prime}$ the projections of $\mathbf{u}_{l l}^{\prime}$ and $\mathbf{u}_{l u}^{\prime}$, respectively, onto $-\nabla \bar{\psi}_{u}$, estimated at the lower-layer cyclone center. 
Figures $9 \mathrm{~b}$ and $9 \mathrm{c}$ show the time evolution of $v_{l l, \perp}^{\prime}$ and $v_{l u, \perp}^{\prime}$. The cyclone velocities induced by the lower-layer $\mathrm{PV}, v_{l l, \perp}^{\prime}$, are negative in both cases and similar to each other until $t=18 \mathrm{~h}$ (Fig. 9b). The negative sign of $v_{l l, \perp}^{\prime}$ can be easily understood when looking at the anomalous PV (Fig. 7): in the lower layer, the anticyclone mainly develops to the southwest of the cyclone and tends to advect it southward across the $\bar{\psi}_{u}$ isolines (see the dashed lines in Fig. 7). Concerning the cyclone velocity induced by the upper-layer $v_{l u, \perp}^{\prime}$, there is a difference in speed between both cases after $9 \mathrm{~h}$ (Fig. 9c), as was also observed in the total speed $v_{l, \perp}^{\prime}$ (Fig. 9a). Therefore, the faster motion of the deformed lower-layer cyclone is attributed to $v_{l u, \perp}^{\prime}$ (Fig. 9c), that is to say, to the upper-layer disturbances instead of the lower-layer disturbances.

To examine the separate effect of the upper-layer cyclone and anticyclone on the lower-layer cyclone motion, the velocity field $\mathbf{u}_{l u}^{\prime}$ is decomposed into $\mathbf{v}_{l u}^{\prime \text { Cup }}$, the velocity induced by the upper-layer positive $\mathrm{PV}$ [i.e., $q_{u}^{\prime} \times$ $\left.H\left(q_{u}^{\prime}\right)\right]$, and $\mathbf{v}_{l u}^{\prime \text { ACup }}$, the velocity induced by the negative $\mathrm{PV}$ in the upper layer [i.e., $q_{u}^{\prime} \times H\left(-q_{u}^{\prime}\right)$, where $H(\cdot)$ is the Heaviside function]. The projections of $\mathbf{v}_{l u}^{\prime \text { Cup }}$ and $\mathbf{v}_{l u}^{\text {'ACup }}$ onto $-\nabla \bar{\psi}_{u}$ are noted as $v_{l u, \perp}^{\prime \text { Cup }}$ and $v_{l u, \perp}^{\prime \text { ACup }}$, respectively.

Figure $9 \mathrm{~d}$ shows the time evolution of both $v_{l u, \perp}^{\prime \text { Cup }}$

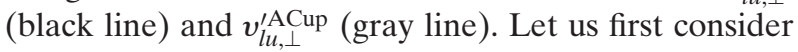
the case of the cyclone initialized in the $\Delta>0$ region (solid lines). Both the upper-layer cyclone and anticyclone lead to the jet crossing by the lower-layer cyclone (see the positive sign of both quantities). More precisely, the contribution of the upper-layer cyclone dominates over that of the upper-layer anticyclone during the first $9 \mathrm{~h}$, in agreement with the stronger amplitude of the upper-layer cyclone than that of the upper-layer anticyclone. Note that $v_{l u, \perp}^{\prime A C u p}$ is not initially zero. Indeed, there exists an initial upper-layer anticyclone in terms of PV because the initialization of the perturbations is made in terms of relative vorticity. After $9 \mathrm{~h}$ the effect of the upper-layer anticyclone prevails (cf. the solid gray and black lines in Fig. 9d): the reason is that the upperlayer anticyclone keeps strengthening (Fig. 8c) whereas the upper-layer cyclone keeps weakening (Fig. 8b). A similar result is obtained for the cyclone initialized in the $\Delta<0$ region. The difference of $v_{l u, \perp}^{\prime}$ between the more deformed cyclone and the less deformed one seems to be strictly due to the upper-layer anticyclone (see the

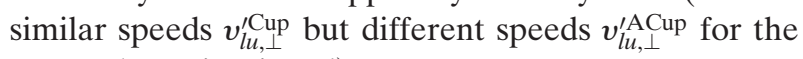
two cyclones in Fig. 9d).

To investigate further the reason why the speed $v_{l u, \perp}^{\text {ACup }}$ is stronger for the most deformed cyclone than for the less deformed one, the modulus of $\mathbf{v}_{l u}^{\prime \text { ACup }}$ (denoted as $\left.\left\|\mathbf{v}_{l u}^{\prime \text { ACup }}\right\|\right)$ and the angle of $\mathbf{v}_{l u}^{\prime \text { ACup }}$ with respect to $-\nabla \bar{\psi}_{u}$ (denoted $\vartheta$ ) are introduced. Figures $9 \mathrm{e}$ and 9 f show the time evolution of $\left\|\mathbf{v}_{l u}^{\prime \text { ACup }}\right\|$ and $\vartheta$, respectively. The modulus $\left\|\mathbf{v}_{l u}^{\prime \mathrm{ACup}}\right\|$ is greater and $\vartheta$ is smaller for the most deformed cyclone than for the less deformed one after $9 \mathrm{~h}$. Thus, both parameters explain the more rapid crossjet motion of the most deformed cyclone compared to the less deformed one. The role played by $\vartheta$ can be interpreted by looking at the location and shape of the upper-layer anticyclone. Figure $6 \mathrm{c}$ shows that the upperlayer anticyclone associated with the quasi-isotropic upper-layer cyclone tends to curl around it. Indeed, the anticyclone being weaker than the cyclone, it tends to be advected and deformed along the southeast-northwest direction by the cyclone and to sprawl around it. The southeast-northwest tilted anticyclone can explain the large values of $\vartheta$ (after $9 \mathrm{~h}$ in Fig. 9f). On the contrary, Fig. $6 \mathrm{~d}$ shows that the upper-layer anticyclone remains east-northeast of the deformed upper-layer cyclone; it is stronger than the cyclone and is thus less deformed by it. On its western side, its isolines being almost aligned with the cross-streamlines direction, it leads to weak values of $\vartheta$ (Fig. 9f). This renders the anticyclone in that case more efficient in pushing the lower-layer cyclone across the basic-state isolines.

The two previous simulations were initialized with a lower-layer cyclone located at distance $d=750 \mathrm{~km}$ to the south of the jet axis, in a negative and positive effective deformation region. To study the role of the initial distance of the cyclones relative to the jet axis, other simulations were made with cyclones initialized at $d=625 \mathrm{~km}$ and $d=1000 \mathrm{~km}$ at the same longitudes. The jet-crossing times are 14.5 and $11.5 \mathrm{~h}$ for cyclones initialized at $d=625 \mathrm{~km}$ in the $\Delta<0$ and $\Delta>0$ regions, respectively, and 33.5 and $21.5 \mathrm{~h}$ at $d=1000 \mathrm{~km}$. The cyclone initialized near the jet core and in the region of positive $\Delta$ has the shortest jet-crossing time, as expected from the previous results. Figures $10 \mathrm{a}$ and $10 \mathrm{~b}$ show respectively the time evolution of the minimum (in absolute value) of the upper-layer perturbation relative vorticity and the time evolution of the speed $v_{l u, \perp}^{\prime}$. For each distance, the anticyclones and the velocity $v_{l u, \perp}^{\prime}$ are shown to be larger in the $\Delta>0$ case than in the $\Delta<0$ case. This is similar to the results obtained in the simulations with cyclones initialized at $d=750 \mathrm{~km}$ (see Figs. $8 \mathrm{c}$ and $9 \mathrm{c}$ ). Moreover, Fig. 10a shows that the upper-layer anticyclone is stronger for cyclones initialized near the jet core than for those initialized farther away. As the largescale PV gradient in the upper layer is stronger near the jet core (see Fig. 4b), a more efficient energy dispersion occurs, leading to more intense upper-layer anticyclones. The smaller anticyclone and a less favorable tilt $\vartheta$ (not shown) are responsible for a smaller velocity $v_{l u, \perp}^{\prime}$ during the first $12 \mathrm{~h}$ for cyclones far from the jet core (Fig. 10b).

Results were found to be robust by doubling the amplitude $A_{k}$ of the initial cyclones and for other values of 
a) Intensity of the upper anticyclone

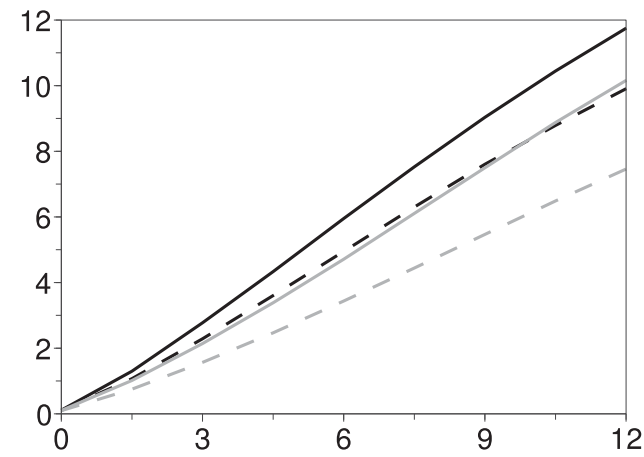

b) Cross-jet speed

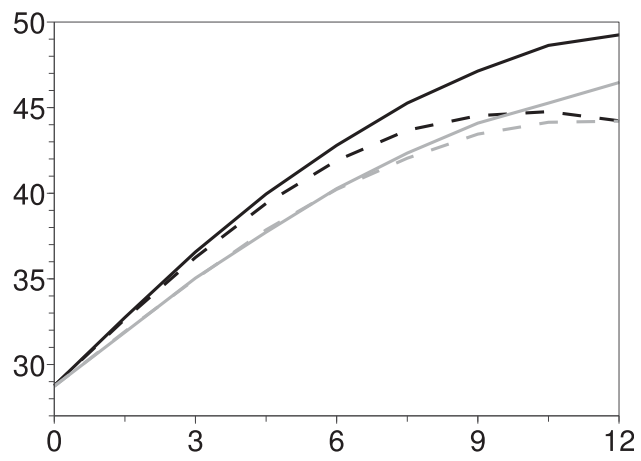

FIG. 10. Evolution with time (h) of (a) the minimum, in absolute value, of the upper-layer relative vorticity $\left(10^{-5} \mathrm{~s}^{-1}\right)$ and (b) $v_{l u, \perp}^{\prime}\left(\mathrm{m} \mathrm{s}^{-1}\right)$ for disturbances initially located at $d=625 \mathrm{~km}$ (black) or $d=1000 \mathrm{~km}$ (gray) in the negative (dashed) and positive (solid) effective deformation regions.

the initial distance $d$. Note finally that changing the parameters of the large-scale jet $\epsilon$ and $\alpha$ [see Eq. (8) and Table 1] and the definition of the initial cyclones (for instance in terms of PV) lead to similar results as well.

\section{c. Confirmation through statistical analysis}

A statistical study based on more than a thousand simulations was made to confirm the previously underlined mechanism. The model is successively initialized with 256 disturbances located to the south of the jet core, each of them being defined as in section $2 b$. The abscissas $x_{0}$ of the lower-layer perturbation centers vary between -8000 and $8000 \mathrm{~km}$ every $62.5 \mathrm{~km}$. The corresponding ordinates $y_{0}$ are defined as $y_{0}=\epsilon \sin \left(K x_{0}-\right.$ $\pi / 4)-d$. The distance to the jet core is denoted as $d$ and varies between 375 and $1000 \mathrm{~km}$ every $125 \mathrm{~km}$. The location of the associated upper-layer perturbation (upstream of the lower-layer perturbation) is calculated as explained in section $2 \mathrm{~b}$. This set of experiments leads to $256 \times 6=1536$ trajectories. Since differences between cases are only visible after a few hours, we will examine time averaged quantities between 9 and $18 \mathrm{~h}$. Figure 11a is a scatterplot of the averaged aspect ratio of the lowerlayer cyclones as a function of the averaged effective deformation $\Delta$, both integrated along the cyclones trajectory. The symbols are associated with different initial distances $d$. For each distance, the correlation between the elongation of the lower-layer cyclones and the largescale effective deformation field is clear, as expected from the role of $\Delta$, except for disturbances very close to the jet axis, such as at $d=375 \mathrm{~km}$ and $d=500 \mathrm{~km}$. In those cases, the elongation of the surface cyclones is more complex to interpret because they cross the jet between 9 and $18 \mathrm{~h}$ and thus evolve in an inhomogeneous deformation field. Indeed, during the jetcrossing phase, the effective deformation $\Delta$ can change its sign, or the dilatation axes can suddenly change their orientation.

The intensities of the lower-layer cyclone, upper-layer cyclone, and upper-layer anticyclone at $t=18 \mathrm{~h}$ are represented in Figs. 11b-d, respectively, as a function of the averaged aspect ratio of the lower-layer cyclone. The amplitude of the lower-layer cyclone is almost independent of the averaged aspect ratio and of the initial distance to the jet core (Fig. 11b). Moreover, the upperlayer and lower-layer cyclones initialized at $d=1000 \mathrm{~km}$ (i.e., far from the jet core) have comparable amplitudes (cf. Figs. 11b and 11c) close to the initial value $(1.5 \times$ $\left.10^{-4} \mathrm{~s}^{-1}\right)$. Indeed, the upper-layer and lower-layer PV gradient having almost the same amplitude at this distance (Fig. 4b), the upper-layer and lower-layer vortices lose the same energy by Rossby wave emission. For distances less than $d=1000 \mathrm{~km}$, the upper-layer cyclone has systematically a smaller amplitude than the lower-layer cyclone (cf. Figs. 11b and 11c) and this is all the more true when $d$ decreases and $r$ increases. On the contrary, the upper-layer anticyclone is stronger for more elongated cyclones or closer to the jet axis (Fig. 11d). Note that the upper-layer anticyclone is less sensitive to the initial distance $d$ than the upper-layer cyclone (Figs. 11c,d). It can be explained by the fact that the cyclone keeps losing energy by emitting Rossby waves, whereas the anticyclone gets energy from the initial cyclone and loses energy at the benefit of a more downstream secondary cyclone at the same time (see Figs. 7e,f). Thus, energy dispersion has two opposite effects on the anticyclone, which makes it less sensitive to the upper-layer PV gradient. To summarize Fig. 11, the evolution of the amplitude of both upper-layer cyclonic and anticyclonic structures with the aspect ratio and the distance is consistent with the results obtained by Oruba et al. (2012) in a barotropic context and they 
(a)

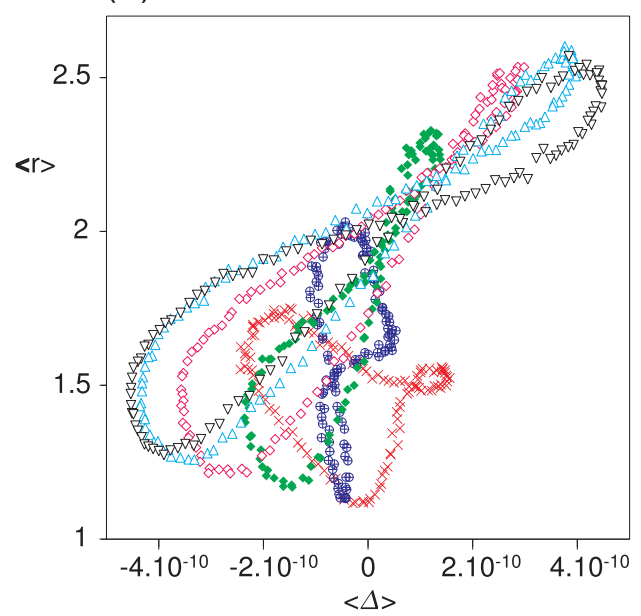

(c)

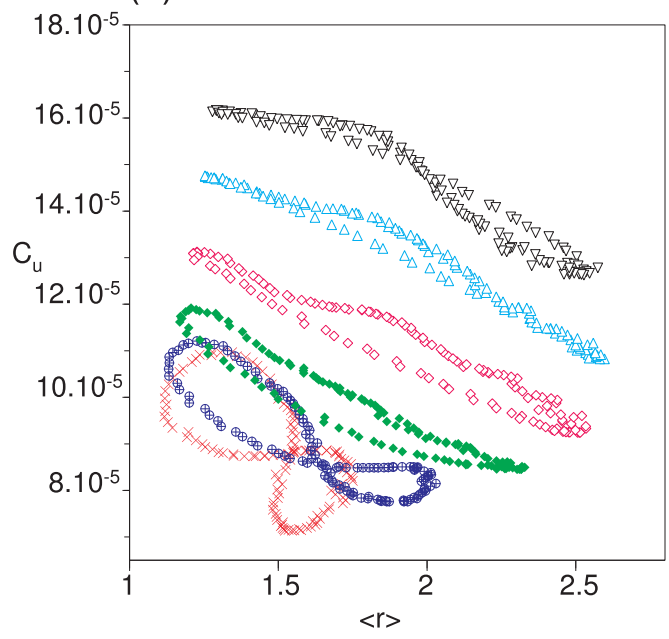

(b)

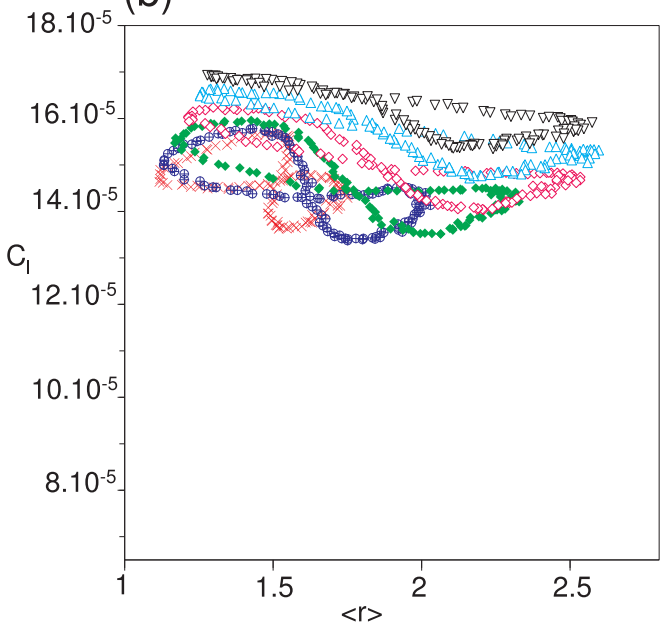

(d)

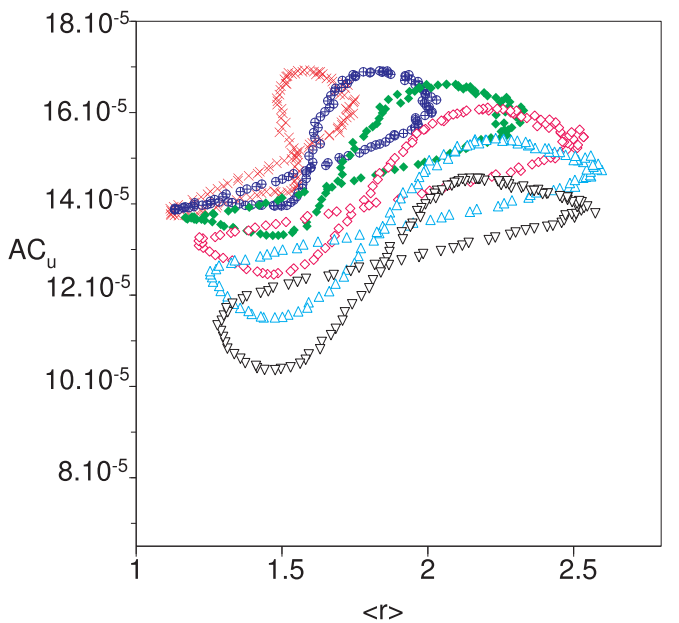

FIG. 11. (a) Scatterplot of the aspect ratio $r$ of the lower-layer cyclone averaged between 9 and $18 \mathrm{~h}$ vs the average of $\Delta\left(\mathrm{s}^{-2}\right)$. Scatterplots of the maximum of the relative vorticity $\left(\mathrm{s}^{-1}\right)$ field in the (b) lower and (c) upper layers and (d) of the minimum of the upper-layer relative vorticity field (absolute value), all at $t=18 \mathrm{~h}$, as a function of the averaged $r: d=375 \mathrm{~km}$ (crosses, red), $d=500 \mathrm{~km}$ (circles, blue), $d=625 \mathrm{~km}$ (lozenges, green), $d=750 \mathrm{~km}$ (diamonds, pink), $d=875 \mathrm{~km}$ (triangles, cyan), and $d=1000 \mathrm{~km}$ (reverse triangles, black).

corroborate the observations of the previous section. The emission of Rossby waves reinforcing the upperlayer anticyclone depends on the amplitude of the largescale PV gradient in the upper layer (which decreases with the distance, see Fig. 4b) and on the stretching of the cyclone (which increases with $\Delta$, see Figs. 11a,d).

Figure 12a is a scatterplot of the averaged cross-jet speed $v_{l, \perp}^{\prime}$ of the lower-layer cyclones as a function of their averaged aspect ratio. It shows that, for each distance, $v_{l, \perp}^{\prime}$ increases with the averaged aspect ratio of the lower-layer cyclone. Furthermore, as shown in Fig. 12b by considering all distances, $v_{l, \perp}^{\prime}$ increases with the integrated large-scale PV gradient. It confirms the key roles played by the barotropic PV gradient and the elongation of the cyclones in the cross-jet motion.
Figures $12 \mathrm{c}$ and $12 \mathrm{~d}$ are scatterplots of the averaged cross-jet speeds $v_{l u, \perp}^{\prime}$ and $v_{l l, \perp}^{\prime}$ as a function of the averaged $v_{l, \perp}^{\prime}$. The cross-jet speeds $v_{l, \perp}^{\prime}$ and $v_{l u, \perp}^{\prime}$ are positively correlated for each distance and it is also true when considering all distances (Fig. 12c). However, for each distance, the cross-jet speed $v_{l l, \perp}^{\prime}$ is anticorrelated with $v_{l, \perp}^{\prime}$ since it decreases while $v_{l, \perp}^{\prime}$ increases (Fig. 12d). We conclude that the advection of the lower-layer cyclone by the upper-layer disturbances, and not by the lowerlayer ones, leads to the jet crossing by the lower-layer cyclones.

The separate roles of the upper-layer anticyclone and cyclone in the cross-jet speed of the lower-layer cyclone can now be investigated. Figures $13 \mathrm{a}$ and $13 \mathrm{~b}$ are scatterplots of the averaged $v_{l u, \perp}^{\prime \text { ACup }}$ and $v_{l u, \perp}^{\prime \text { Cup }}$, respectively, as 
(a)

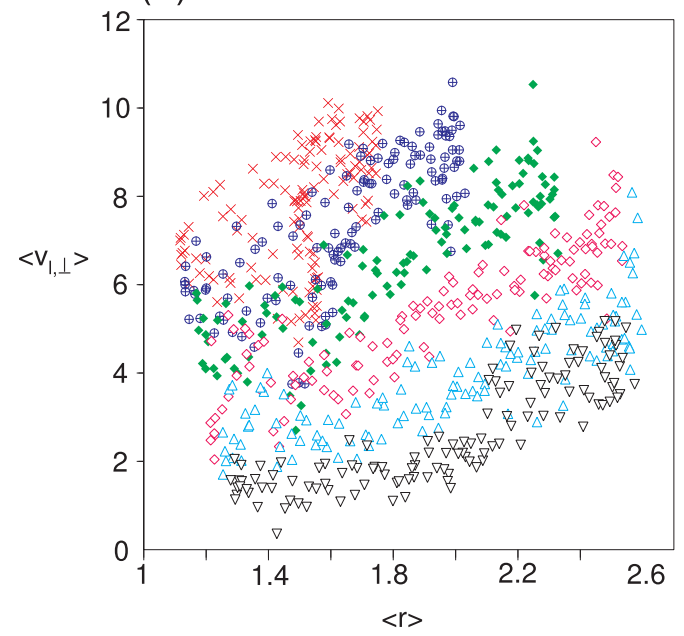

(c)

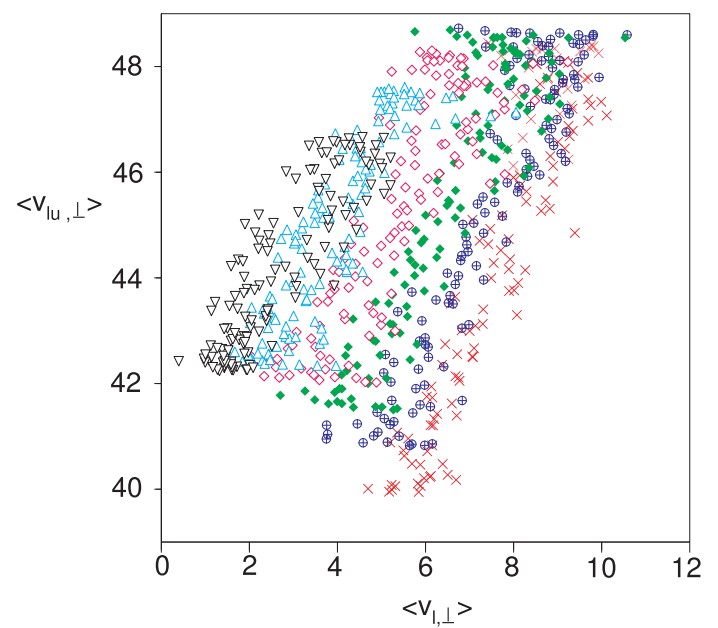

(b)

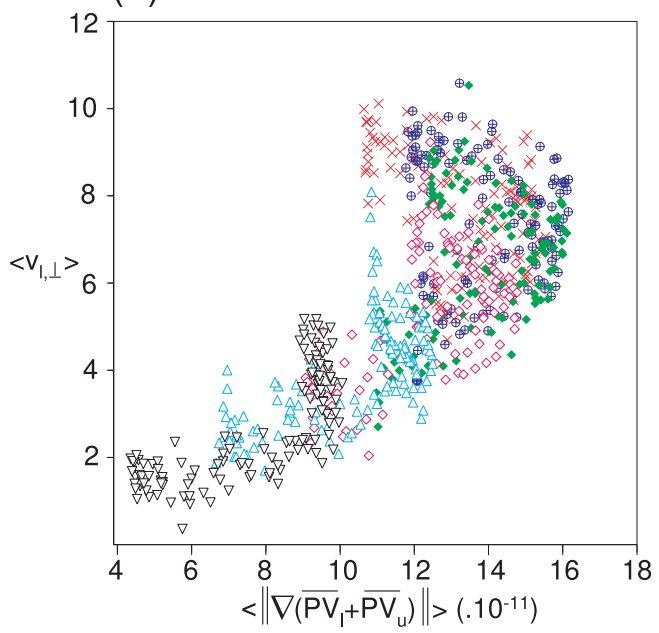

(d)

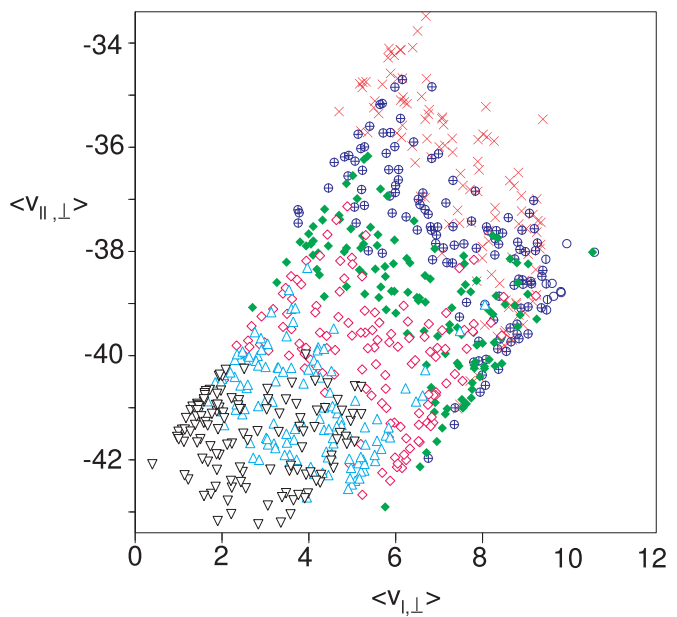

FIG. 12. Scatterplot of $v_{l, \perp}^{\prime}\left(\mathrm{m} \mathrm{s}^{-1}\right)$ (a) vs $r$ and (b) vs the large-scale barotropic PV gradient $\left(\mathrm{m}^{-1} \mathrm{~s}^{-1}\right)$. Scatterplots of (c) $v_{l u, \perp}^{\prime}$ and (d) $v_{l l, \perp}^{\prime}$ vs $v_{l, \perp}^{\prime}\left(\mathrm{m} \mathrm{s}^{-1}\right)$. All averaged between 9 and $18 \mathrm{~h}$. Symbols as in Fig. 11.

a function of the averaged $v_{l \perp}^{\prime}$. For each distance, there is a clear correlation between $v_{l u, \perp}^{\prime \text { ACup }}$ and $v_{l, \perp}^{\prime}$ (Fig. 13a) and between $v_{l u, \perp}^{\prime \text { Cup }}$ and $v_{l, \perp}^{\prime}$ (Fig. 13b). When all distances to the jet and all cyclones are taken into account, the correlation between $v_{l u, \perp}^{\prime \text { ACup }}$ and $v_{l, \perp}^{\prime}$ is well established, whereas the correlation between $v_{l u, \perp}^{\prime \text { Cup }}$ and $v_{l, \perp}^{\prime}$ is less clear. Note that the decrease of $v_{l u, \perp}^{\prime \text { Cup }}$ when $d$ decreases (see Fig. 13b when all distances are taken into account) is due to the stronger energy dispersion closer to the jet. Indeed, the closer to the jet axis, the greater the upperlayer meridional PV gradient (Fig. 4b), so the faster the decrease in the upper-layer cyclone amplitude. In agreement with the previous section, we deduce that the upper-layer anticyclone is responsible for the jet crossing by the lower-layer cyclones and not the upper-layer cyclone.
Figures $13 \mathrm{c}$ and $13 \mathrm{~d}$ are scatterplots of the averaged $\left\|\mathbf{v}_{l u}^{\prime \text { ACup }}\right\|$ and $\cos \vartheta$, respectively, as a function of the averaged $v_{l, \perp}^{\prime}$. All distances taken together, the correlation of $v_{l, \perp}^{\prime}$ with $\cos \vartheta$ seems better than that with $\left\|\mathbf{v}_{l u}^{\prime \text { ACup }}\right\|$. For each distance, $\cos \vartheta$ increases with $v_{l, \perp}^{\prime}$ (Fig. 13d) whereas there is no clear correlation between $\left\|\mathbf{v}_{l u}^{\prime \text { ACup }}\right\|$ and $v_{l, \perp}^{\prime}$ for the large distances at $d=875$ and $1000 \mathrm{~km}$ (Fig. 13c). Thus, both the modulus and the direction

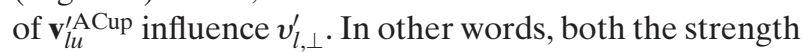
of the upper-layer anticyclone (to which $\left\|\mathbf{v}_{l u}^{\prime A C u p}\right\|$ is linked) and its location and shape (to which the diagnosis $\vartheta$ is linked) play a role into the jet crossing by the lowerlayer cyclone. Nevertheless, as explained in the previous section, the location and the shape of the upper-layer anticyclone depend on its strength. Indeed, a strong upper-layer anticyclone forms a more coherent dipole 
(a)

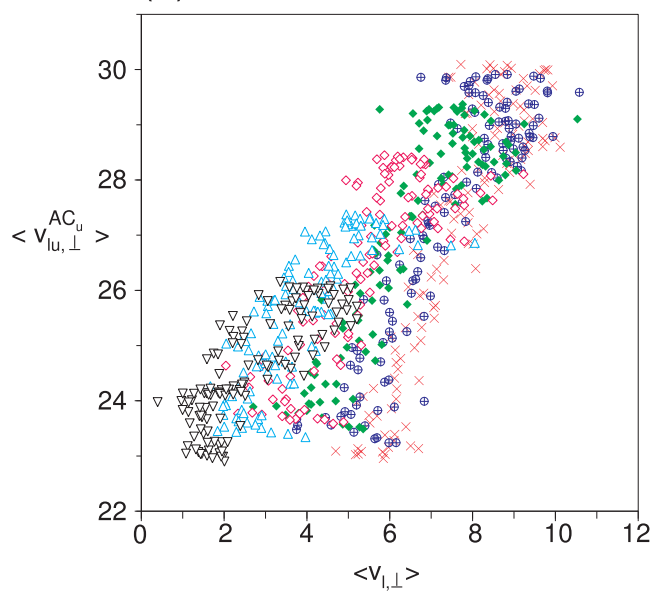

(c)

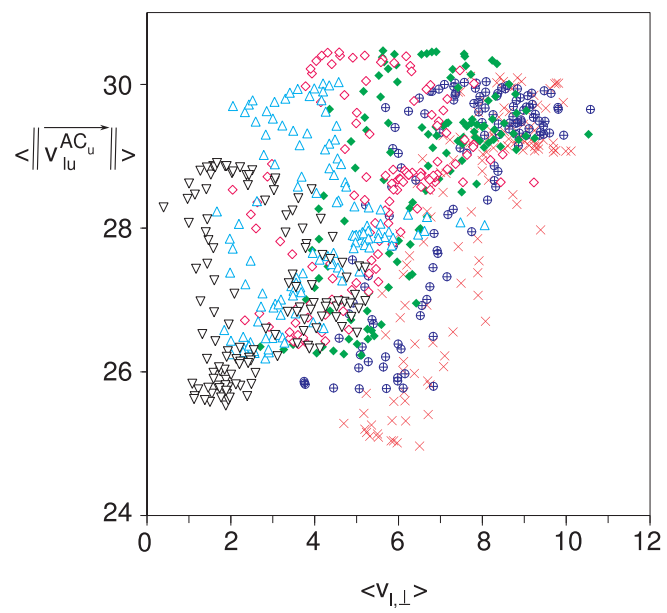

(b)

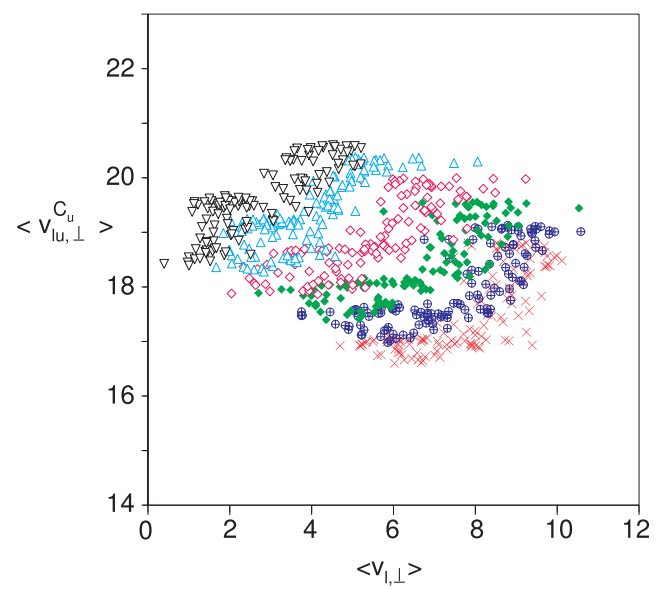

(d)

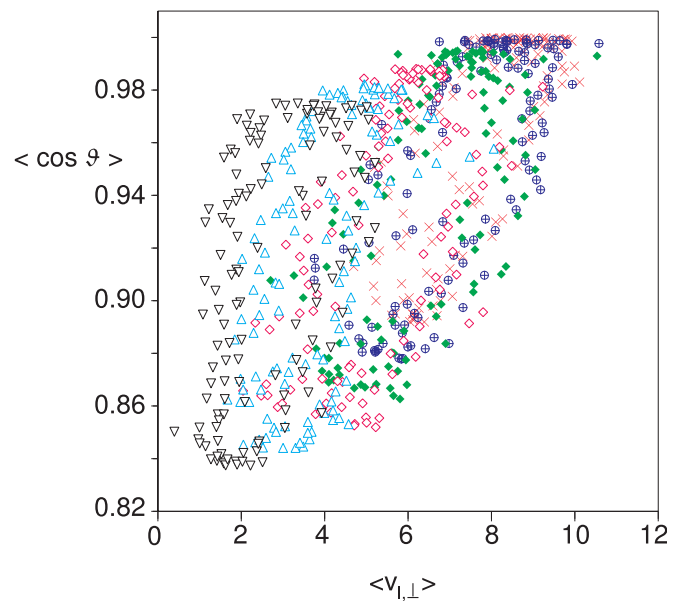

FIG. 13. Scatterplot of (a) $v_{l u, \perp}^{\prime \mathrm{ACup}}$, (b) $v_{l u, \perp}^{\prime \text { Cup }}$, (c) $\left\|\mathbf{v}_{l u}^{\prime \mathrm{ACup}}\right\|$, and (d) $\cos \vartheta \mathrm{vs} v_{l, \perp}^{\prime}\left(\mathrm{m} \mathrm{s}^{-1}\right)$. All averaged between 9 and $18 \mathrm{~h}$. Symbols as in Fig. 11.

with the upper-layer cyclone and remains to the northeast by comparison with a weak upper-layer anticyclone that curls around the upper-layer cyclone, leading to a less favorable configuration for the crossjet motion.

\section{Conclusions and discussion}

We have investigated the role of the horizontal deformation and nonlinearities on the trajectory of a surface cyclonic eddy in the presence of a baroclinically unstable flow using a two-layer quasigeostrophic model on the beta plane. Various numerical experiments have been performed with different background jets and localized upper-layer and lower-layer cyclonic anomalies to the south of the jet in a configuration favorable to their baroclinic interaction. It was shown that the cyclone trajectory can be explained by the theory of beta drift generalized to a baroclinic atmosphere.

In the case of a horizontally uniform zonal basic flow, the large-scale vertically averaged basic-state PV gradient plays a role in the motion of the surface cyclones, as initially described by Gilet et al. (2009). This result was here explained by noting that a positive vertically averaged basic-state PV gradient in a baroclinic flow leads to an asymmetry in the dispersion of Rossby waves. Indeed, as the positive upper-layer PV gradient is stronger than the negative lower-layer PV gradient in absolute value, the upper-layer eastward energy radiation is stronger than the lower-layer westward energy radiation. It induces the growth of a stronger anticyclone in the upper layer than in the lower one. This upperlayer anticyclone forms a dipole with the associated upper-layer cyclone, which is responsible for the poleward 
a) Perturbation potential energy

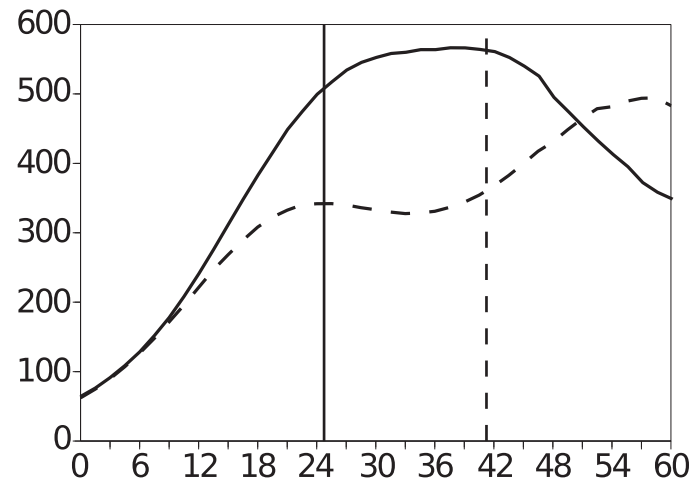

b) Lower-layer perturbation kinetic energy

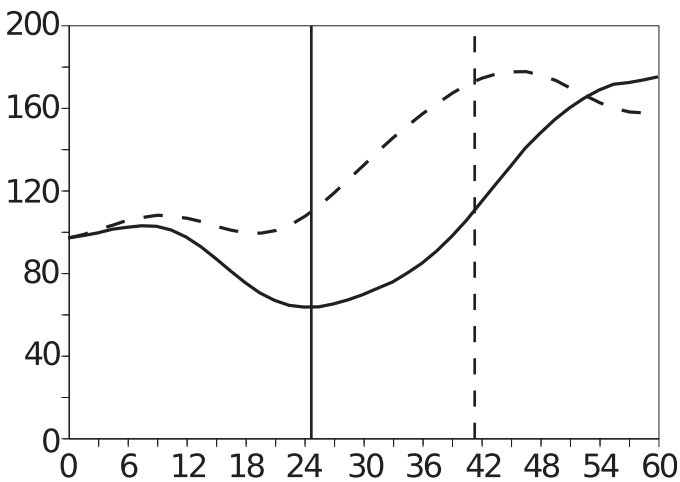

FIG. 14. Evolution with time (h) of (a) the perturbation potential energy and (b) the lower-layer perturbation kinetic energy $\left(\mathrm{m}^{2} \mathrm{~s}^{-2}\right)$ for disturbances initially located in negative (dashed) and positive (solid) effective deformation regions. The energy budget is averaged over a region centered at the maximum of the lower-layer perturbation relative vorticity. Vertical lines indicate the crossing time.

motion of the lower-layer cyclone through a nonlinear effect. This constitutes a dynamical interpretation of the beta-drift mechanism in a baroclinic context and provides a potential vorticity rationale for the idealized cases of Gilet et al. (2009) and the motion of the European storm Xynthia (Rivière et al. 2012).

Then the case of a meandering baroclinically unstable westerly jet has been examined to include horizontal deformation effects. A comparison between cyclonic anomalies evolving in a deformation-dominated environment and a rotation-dominated environment was performed to highlight how the deformation modulates the cross-jet motion of surface cyclones. Cyclonic anomalies are stretched by the deformation-dominated environment, whereas they tend to remain quasi isotropic in the rotation-dominated one. The more stretched surface cyclone is associated with a stronger upper-layer anticyclone and moves perpendicularly to the jet faster than the less stretched one. Both intensity and location of the upperlayer anticyclone explain the distinct cross-jet speeds. The stronger upper-layer anticyclone in the stretched case stays more to the northeast of the upper-layer cyclone than in the less stretched case. This spatial configuration is more efficient to advect the surface cyclone across the jet. These results are consistent with the results of Oruba et al. (2012) obtained in a barotropic context.

These first conclusions, obtained by comparing the cases of a stretched and a nonstretched surface cyclone, were confirmed by a statistical study based on hundreds of simulations with cyclones initialized at different locations south of the jet. It corroborates the main role played by the barotropic large-scale PV gradient. The stronger the gradient, the stronger the upper-layer anticyclone and the quicker the cross-jet motion. The modulation of the effect of the barotropic large-scale PV gradient by deformation effects was also confirmed since a high correlation between the stretching of surface cyclones, the strength and the location of the associated upper-layer anticyclone, and the cross-jet speed of surface cyclones was found.

The lower-layer cyclone and the upper-layer anticyclone observed in our study could be linked to the baroclinic dipole (heton) in the 2.5-layer model of Vandermeirsch et al. (2003) formed by the lower-layer negative anomaly and the cyclonic meander of the upper-layer jet. But, in their study, as well as in Vandermeirsch et al. (2001) or Gilet et al. (2009), the large-scale jet is zonal, which does not include evidence of the role of horizontal deformation, contrary to the present study where the large-scale flow has a complex large-scale horizontal deformation field (baroclinic meandering jet).

Our idealized study allows reproducing to some extent the behavior of the FASTEX IOP17 and "Xynthia" storms. Indeed, the setting of a meandering large-scale jet and of disturbances initialized south of the jet, upstream of a region of positive effective deformation, looks like the situation of these two real cases prior to the jet-crossing phase. In particular, the cyclones initialized in the positive $\Delta$ region cross the jet axis close to the saddle point of the effective deformation field like the two real storms. The energy budget shown in Fig. 14 also corroborates our finding. Since the upper-layer cyclone is initially upstream of the lower-layer one, the perturbation streamlines tilt against the background vertical shear, leading to a strong potential energy extraction from the basic flow by the disturbances (Fig. 14a). The more deformed cyclone more efficiently extracts energy than the less stretched one because of the 
well-maintained tilt with height before the jet-crossing phase. The lower-layer kinetic energy presents several distinct stages. The kinetic energy of the most elongated surface cyclone slightly increases during the first hours, and then decreases before rapidly increasing after the jet crossing (see Fig. 14b, solid line). This energetic life cycle, characterized by a decay phase before the jet crossing and a regeneration stage just after, is similar to that of the FASTEX IOP17 [cf. Fig. 14b herein and Fig. 10d of Rivière and Joly (2006a)]. The various energy conversion rates that explain these kinetic-energy fluctuations are the same in the idealized and real cases (not shown). On the contrary, the deepening just after the jet crossing does not occur for the less deformed cyclone case (see Fig. 14b, dashed line). To conclude, a significant part of the behavior of a real cyclone can be reproduced if the large-scale flow and its related deformation in which the cyclones evolve are well modeled.

The sensitivity of surface cyclone trajectory to the large-scale deformation field, revealed by the present study, may constitute a first step toward an understanding of the existence of preferential regions for jet-crossing phases of real surface depressions, and of the particular role played by barotropic critical regions (saddle point of the effective deformation field) in depression deepening (Rivière and Joly 2006a). This aspect was investigated in a barotropic context by Oruba et al. (2012) and in the present idealized study (not shown), but results were not conclusive. Adding a zonally confined component to the present meandering large-scale jet might improve this aspect as the flow would be more realistic. One can think that preferential regions might be more pronounced when adding jet-exit and jet-entrance regions to the present meandering large-scale jet. It would allow, on the one side, increasing deformation effects within a more realistic environment and, on the other side, confronting our mechanism to other ones such as those involving transverse ageostrophic circulations in the presence of jet-exit regions (Uccelini 1990).

Finally, diabatic effects may also potentially play a role in the crossing of the jet stream by surface cyclones. It is well known that the amplitude of the upper-level ridge downstream of the surface cyclone is reinforced by diabatic effects (Grams et al. 2011), which was found to play a crucial role in the motion of surface cyclones as shown in the present paper.

Acknowledgments. We acknowledge financial support from INSU/LEFE-IDAO project EPIGONE. We dedicate this work to the memory of Bach Lien Hua who was the thesis supervisor of GL and GR and has introduced some new concepts associated with horizontal deformation.

\section{REFERENCES}

Baehr, C., B. Pouponneau, F. Ayrault, and A. Joly, 1999: Dynamical characterization and summary of the FASTEX cyclogenesis cases. Quart. J. Roy. Meteor. Soc., 125, 3469 3494.

Blackmon, M. L., J. M. Wallace, N. C. Lau, and S. L. Mullen, 1977: An observational study of the Northern Hemisphere wintertime circulation. J. Atmos. Sci., 34, 1040-1053.

Bretherton, F. P., 1966: Baroclinic instability and the short wavelength cutoff in terms of potential vorticity. Quart. J. Roy. Meteor. Soc., 92, 335-345.

Cohen, R., and D. Schultz, 2005: Contraction rate and its relationship to frontogenesis, the Lyapunov exponent, fluid trapping, and airstream boundaries. Mon. Wea. Rev., 133, 1353-1369.

Colucci, S. J., 1985: Explosive cyclogenesis and large-scale circulation changes: Implications for atmospheric blocking. J. Atmos. Sci., 42, 2701-2717.

Davies, H. C., and C. H. Bishop, 1994: Eady edge waves and rapid development. J. Atmos. Sci., 51, 1930-1946.

_ C. Schär, and H. Wernli, 1991: The palette of fronts and cyclones within a baroclinic wave development. J. Atmos. Sci., 48, 1666-1689.

Fink, A. H., T. Brücher, V. Ermert, A. Krüger, and J. G. Pinto, 2009: The European storm Kyrill in January 2007: Synoptic evolution, meteorological impacts and some considerations with respect to climate change. Nat. Hazards Earth Syst. Sci., 9, 405-423.

Flierl, G. R., 1977: The application of linear quasigeostrophic dynamics to Gulf Stream Rings. J. Phys. Oceanogr., 7, 365-379.

Gilet, J.-B., M. Plu, and G. Rivière, 2009: Nonlinear baroclinic dynamics of a surface cyclone crossing a zonal jet. J. Atmos. Sci., 66, 3021-3041.

Grams, C., and Coauthors, 2011: The key role of diabatic processes in modifying the upper-tropospheric wave guide: A North Atlantic case-study. Quart. J. Roy. Meteor. Soc., 137, 2174 2193.

Heifetz, E., C. H. Bishop, B. J. Hoskins, and J. Methven, 2004: The counter-propagating Rossby-wave perspective on baroclinic instability. I: Mathematical basis. Quart. J. Roy. Meteor. Soc., 130, 211-231.

Holland, G. J., 1983: Tropical cyclone motion: Environmental interaction plus a beta effect. J. Atmos. Sci., 40, 328-342.

Hoskins, B. J., and K. I. Hodges, 2002: New perspectives on the Northern Hemisphere winter storm tracks. J. Atmos. Sci., 59, 1041-1061.

Joly, A., and Coauthors, 1999: Overview of the field phase of the Fronts and Atlantic Storm-Track EXperiment (FASTEX) project. Quart. J. Roy. Meteor. Soc., 125, 3131-3163.

Jones, S. C., 1995: The evolution of vortices in vertical shear. I: Initially barotropic vortices. Quart. J. Roy. Meteor. Soc., 121, 821-851.

_ 2000a: The evolution of vortices in vertical shear. III: Baroclinic vortices. Quart. J. Roy. Meteor. Soc., 126, 31613185 .

- 2000b: The evolution of vortices in vertical shear. II: Largescale asymmetries. Quart. J. Roy. Meteor. Soc., 126, 31373159.

Kida, S., 1981: Motion of an elliptic vortex in a uniform shear flow. J. Phys. Soc. Japan, 50, 3517-3520.

Lacasce, J. H., 1998: A geostrophic vortex over a slope. J. Phys. Oceanogr., 28, 2362-2381. 
Lapeyre, G., P. Klein, and B. L. Hua, 1999: Does the tracer gradient vector align with the strain eigenvectors in $2 \mathrm{D}$ turbulence? Phys. Fluids, 11, 3729-3737.

McWilliams, J., and G. Flierl, 1979: On the evolution of isolated nonlinear vortices. J. Phys. Oceanogr., 9, 1155-1182.

Methven, J., E. Heifetz, B. Hoskins, and C. Bishop, 2005a: The counter-propagating Rossby-wave perspective on baroclinic instability. Part III: Primitive-equation disturbances on the sphere. Quart. J. Roy. Meteor. Soc., 131, 1393-1424.

$\longrightarrow$, B. Hoskins, E. Heifetz, and C. Bishop, 2005b: The counterpropagating Rossby-wave perspective on baroclinic instability. Part IV: Nonlinear life cycles. Quart. J. Roy. Meteor. Soc., 131, 1425-1440.

Mied, R. P., and G. J. Lindemann, 1979: The propagation and evolution of cyclonic Gulf Stream rings. J. Phys. Oceanogr., 9, 1183-1206.

Morel, Y. G., 1995: The influence of an upper thermocline current on intrathermocline eddies. J. Phys. Oceanogr., 25, 3247-3252.

_ and J. C. McWilliams, 1997: Evolution of isolated vortices in the ocean. J. Phys. Oceanogr., 27, 727-748.

Murray, R., and S. Daniels, 1953: Transverse flow at entrance and exit to jet streams. Quart. J. Roy. Meteor. Soc., 79, 236-241.

Namias, J., and P. Clapp, 1949: Confluence theory of the high tropospheric jet stream. J. Meteor., 6, 125-133.

Neu, U., and Coauthors, 2013: IMILAST: A community effort to intercompare extratropical cyclone detection and tracking algorithms. Bull. Amer. Meteor. Soc., 94, 529-547.

Okubo, A., 1970: Horizontal dispersion of floatable particles in the vicinity of velocity singularity such as convergences. Deep-Sea Res., 17, 445-454.

Oruba, L., G. Lapeyre, and G. Rivière, 2012: On the northward motion of midlatitude cyclones in a barotropic meandering jet. J. Atmos. Sci., 69, 1793-1810.

Palmen, E., and C. Newton, 1969: Atmospheric Circulation Systems: Their Structure and Physical Interpretation. International Geophysics Series, Vol. 13, Academic Press, 603 pp.

Pedlosky, J., 1987: Geophysical Fluid Dynamics. Springer Verlag, $636 \mathrm{pp}$.

Phillips, N., 1951: A simple three-dimensional model for the study of large-scale extratropical flow patterns. J. Meteor., 8, 381394.

Reznik, G., and Z. Kizner, 2007: Two-layer quasi-geostrophic singular vortices embedded in a regular flow. Part 2. Steady and unsteady drift of individual vortices on a beta-plane. J. Fluid Mech., 584, 203-223.

Rivière, G., 2008: Barotropic regeneration of upper-level synoptic disturbances in different configurations of the zonal weather regime. J. Atmos. Sci., 65, 3159-3178.

— , and A. Joly, 2006a: Role of the low-frequency deformation field on the explosive growth of extratropical cyclones at the jet exit. Part I: Barotropic critical region. J. Atmos. Sci., 63, 1965-1981.

_ and - 2006b: Role of the low-frequency deformation field on the explosive growth of extratropical cyclones at the jet exit. Part II: Baroclinic critical region. J. Atmos. Sci., 63, 19821995.
_ B. L. Hua, and P. Klein, 2003: Perturbation growth in terms of barotropic alignment properties. Quart. J. Roy. Meteor. Soc., 129, 2613-2635.

—-, P. Arbogast, G. Lapeyre, and K. Maynard, 2012: A potential vorticity perspective on the motion of a mid-latitude winter storm. Geophys. Res. Lett., 39, L12808, doi:10.1029/ 2012GL052440.

Sanders, F., 1993: Upper-level geostrophic diffluence and deepening of surface lows. Wea. Forecasting, 8, 339-344.

Schär, C., and H. Wernli, 1993: Structure and evolution of an isolated semi-geostrophic cyclone. Quart. J. Roy. Meteor. Soc., 119, 57-90.

Schultz, D., D. Keyser, and L. Bosart, 1998: The effect of largescale flow on low-level frontal structure and evolution in midlatitude cyclones. Mon. Wea. Rev., 126, 1767-1791.

Shapiro, L. J., 1992: Hurricane vortex motion and evolution in a three-layer model. J. Atmos. Sci., 49, 140-153.

Simmons, A. J., and B. J. Hoskins, 1978: The life cycles of some nonlinear baroclinic waves. J. Atmos. Sci., 35, 414-432.

- , and -1979 : The downstream and upstream development of unstable baroclinic waves. J. Atmos. Sci., 36, 1239-1254.

Sutyrin, G., and Y. G. Morel, 1997: Intense vortex motion in a stratified fluid on the beta-plane: An analytical theory and its validation. J. Fluid Mech., 336, 203-220.

Uccelini, L. W., 1990: Processes contributing to the rapid development of extratropical cyclones. Extratropical Cyclones: The Erik Palmén Memorial Volume, C. W. Newton and E. O. Holopainen, Eds., Amer. Meteor. Soc, 81-105.

Vandermeirsch, F. O., Y. G. Morel, and G. Sutyrin, 2001: The net advective effect of a vertically sheared current on a coherent vortex. J. Phys. Oceanogr., 31, 2210-2225.

_, X. J. Carton, and Y. G. Morel, 2003: Interaction between an eddy and a zonal jet. Part II. Two-and-a-half-layer model. Dyn. Atmos. Oceans, 36, 271-296.

Vautard, R., 1990: Multiple weather regimes over the North Atlantic: Analysis of precursors and successors. Mon. Wea. Rev., 118, 2056-2081.

Vederman, J., 1954: The life cycles of jet streams and extratropical cyclones. Bull. Amer. Meteor. Soc., 35, 239-244.

Wallace, J., G.-H. Lim, and M. Blackmon, 1988: Relationship between cyclone tracks, anticyclone tracks and baroclinic waveguides. J. Atmos. Sci., 45, 439-462.

Weiss, J., 1981: The dynamics of enstrophy transfer in twodimensional hydrodynamics. Center for Studies of Nonlinear Dynamics, La Jolla Institute Tech. Rep. LJI-TN-121ss, $112 \mathrm{pp}$.

Wernli, H., R. Fehlmann, and D. Luthi, 1998: The effect of barotropic shear on upper-level induced cyclogenesis: Semigeostrophic and primitive equation numerical simulations. J. Atmos. Sci., 55, 2080-2094.

— S. Dirren, M. A. Liniger, and M. Zillig, 2002: Dynamical aspects of the life cycle of the winter storm Lothar (24-26 December 1999). Quart. J. Roy. Meteor. Soc., 128, 405-429.

Wu, C.-C., and K. A. Emanuel, 1993: Interaction of a baroclinic vortex with background shear: Application to hurricane movement. J. Atmos. Sci., 50, 62-76. 